\title{
Variety of scaling behaviors in nanocrystalline plasticity
}

\author{
P. Zhang, ${ }^{1}$ O. U. Salman $\odot,{ }^{2}$ J. Weiss $\odot,{ }^{3}$ and L. Truskinovsky ${ }^{4}$ \\ ${ }^{1}$ State Key Laboratory for Mechanical Behavior of Materials, Xi'an Jiaotong University, Xi'an, 710049, China \\ ${ }^{2}$ CNRS, LSPM UPR3407, Paris Nord Sorbonne Université, 93430, Villetaneuse, France \\ ${ }^{3}$ IsTerre, CNRS/Université Grenoble Alpes, 38401 Grenoble, France \\ ${ }^{4}$ PMMH, CNRS UMR 7636, ESPCI ParisTech, 10 Rue Vauquelin, 75005, Paris, France
}

(Received 16 April 2020; revised 22 June 2020; accepted 3 August 2020; published 28 August 2020)

\begin{abstract}
We address the question of why larger, high-symmetry crystals are mostly weak, ductile, and statistically subcritical, while smaller crystals with the same symmetry are strong, brittle and supercritical. We link it to another question of why intermittent elasto-plastic deformation of submicron crystals features highly unusual size sensitivity of scaling exponents. We use a minimal integer-valued automaton model of crystal plasticity to show that with growing variance of quenched disorder, which can serve in this case as a proxy for increasing size, submicron crystals undergo a crossover from spin-glass marginality to criticality characterizing the second order brittle-to-ductile (BD) transition. We argue that this crossover is behind the nonuniversality of scaling exponents observed in physical and numerical experiments. The nonuniversality emerges only if the quenched disorder is elastically incompatible, and it disappears if the disorder is compatible.
\end{abstract}

DOI: 10.1103/PhysRevE.102.023006

\section{INTRODUCTION}

Considerable research efforts have been recently focused on the study of mechanical properties of submicron crystals [1-5]. It was found that the deformation mechanisms, which we habitually associate with dislocation plasticity, change dramatically once the sample size is reduced below the micrometer range. Strength of such crystals was shown to be size-dependent [6-8], with stress-strain response exhibiting pronounced intermittency and scale invariance over a wide range of scales, independently of crystal symmetry [9-16]. Both measured and computed scaling exponents were shown to feature highly unusual size dependence $[17,18]$.

Moreover, even though plasticity at macroscale is generally associated with ductility, crystal plasticity at submicron scales exhibits major stress drops or strain bursts reminiscent of brittle fracture [5,19-22]. Brittleness, usually attributed to dislocation-free crystals [23], reappears in nanoparticles and nanopillars that seem to be "breaking plastically" by generating a large number of globally correlated dislocations [24,25]. The implied system-size events hinder our ability to control plastic deformation at submicron scale and compromise the reliable functioning of ultra-small machinery [10,11,26-28].

The peculiar properties of submicron crystals can be linked to the scarcity of dislocation sources and easiness of surface annihilation. This limits dislocation storage and inhibits forest hardening, thus reducing dislocation network complexity and promoting highly anisotropic single-slip behavior. The lack of obstacles facilitates the collective behavior, which is ultimately behind intermittency and scaling. Rationalization of the crossover from bulk to surface-dominated plastic flow has emerged recently as one of the main challenges in crystal plasticity.
To illustrate the full spectrum of plastic responses at submicron scale, we decided to characterize them experimentally for a single material. We conducted a set of compression tests on pure Mo submicron pillars, choosing intentionally a "mild," in the sense of Ref. [29], BCC crystal. The main qualitative observation was that larger, dislocation-rich submicron crystals are weak, ductile, and statistically subcritical. In contrast, smaller, dislocation-starved crystals are strong, brittle, and statistically supercritical. One of the aims of this paper is to reproduce the observed behavior using a minimal, analytically transparent model.

The intermittent plastic deformation in crystals was modeled previously using molecular dynamics [30], discrete dislocation dynamics [17,19,31-33], phase field theories [34], and various mesoscopic approaches [35,36]. The results of different simulations are not fully consistent, suggesting that scaling exponents may be covering a broad range of values $[18,37,38]$ and supporting the idea that microplasticity is not a universal critical phenomenon.

Here we show that, rather surprisingly, the use of an oversimplified model of crystal plasticity introduced in Refs. [36,39] allows one to reconcile the existing results while dealing with realistic preparations and avoiding ad hoc assumptions. The main step is the reduction of the plastic flow problem to a computationally effective integer-valued discrete automaton. Despite the simplicity of the ensuing dynamical system, one can account in this way for both short- and longrange elastic interactions, including dislocation nucleation and immobilization. It also allows one to accumulate sufficient statistics, since one can deal in this way with millions of mesoscopic elements and tens of thousands of dislocations.

Our main result is that the nonuniversality of submicron plasticity and the inferred brittleness of ultrasmall crystals can 
be conceptualized as a multistage crossover from spin-glass marginality, characteristic of very small, almost defect-free crystals, to the criticality of larger crystals associated with a brittle-to-ductile (BD) transition.

To simulate the size dependence of scaling exponents in small crystals we assumed that, at least in submicron range, the decreasing variance of the quenched disorder can serve as a proxy for contracting crystal size. Behind this assumption is the idea that the role of surface annihilation of dislocations can be mimicked by the scarcity of external sources required for dislocation nucleation in the bulk. Essentially, we exploit the fact that in small systems, the conventional dislocation nucleation sources are compromised or even disabled by their closeness to the surface.

Despite the rather prototypical nature of such approach, we were able to capture both, qualitatively and quantitatively, the size dependence observed in our experiments on Mo micropillars. Rather remarkably, we reproduced almost exactly the measured value of the critical exponent characterizing the BD transition in such crystals. Along the way, we revealed a fundamental distinction between elastically compatible (local) and elastically incompatible (nonlocal) quenched disorder and showed that the nonuniversality emerges only if the quenched disorder is "nonlocal" and that it disappears if the disorder is "local" as in the conventional random field Ising model (RFIM) [40,41].

It should be mentioned that some closely related results have been previously obtained in the studies of amorphous glasses, also exhibiting brittleness, yield, intermittency, and the BD transition. However, outside the limit of extremely well-annealed glasses (practically unreachable), the amorphous systems remain fundamentally different from crystals. For instance, the quenched disorder in glasses and granular systems is often rather special as it is revealed by the hierarchical structure of their energy landscapes. More generally, amorphous solids lack long-range order, which is behind crystallographic constraints for plastic slip, and which ultimately ensures orientation dependence of the mechanical response. Most importantly, mobile dislocations, that may nucleate, annihilate, and form complex entanglements in crystals, do not exist in amorphous solids [41-44]. As we show in this paper, the existence of an additional structure in crystalline plasticity makes their scaling behavior more nuanced.

The rest of the paper is organized as follows. In Sec. II we present the results of our compression tests on Mo micropillars. We then formulate our computational model in Sec. III. The possibility to use quenched disorder as a proxy for the system size is discussed in detail in Sec. IV. The macroscopic stress-strain response of the system is studied in Sec. V, and in Sec. VI we quantify the fractal structure of the associated plastic strain fields. The disorder dependence of the statistics of avalanches is analyzed in Sec. VII. The related distribution of stability measures is discussed in Sec. VIII. A simple mean-field model, building a bridge between our computational results and the macroscopic parameters used in phenomenological models of crystal plasticity, is presented in Sec. IX. In Sec. X we compare the results for monotone and oscillatory loading and in Sec. XI we compare the effects of "local" and "nonlocal" quenched disorders. Our main results are summarized in Sec. XII.

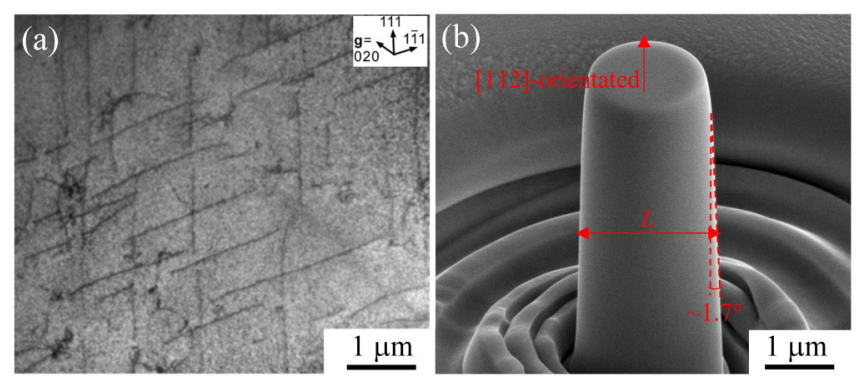

FIG. 1. (a) TEM image showing the grown-in dislocations in the bulk Mo crystal. (b) SEM image of [112]-orientated micropillar before compression.

\section{EXPERIMENT}

A millimeter-sized Mo single crystal was cut from a wellannealed Mo ingot of a high purity (>99.99\%). The initial dislocation structure inside this BCC crystal was characterized by transmission electron microscopy (TEM), showing straight screw dislocations along $\langle 111\rangle$ directions [Fig. 1(a)], with a density $\rho \approx 1.6 \times 10^{12} \mathrm{~m}^{2}$ measured by the line-intercept method. According to these data the estimated equidistant dislocation spacing is $l \sim 1 / \sqrt{\rho} \approx 790 \mathrm{~nm}$.

The [112]-oriented Mo pillars with diameters from $500 \mathrm{~nm}$ to $1500 \mathrm{~nm}$ were fabricated on the electropolished surface of the single bulk crystal using a Ga-operated focused-ion beam. The height-to-diameter ratio of the pillars was kept between $2.5: 1$ and $3: 1$, and the taper was $\sim 1.7^{\circ}$ [Fig. 1(b)]. A nanoindentation system (Hysitron $\mathrm{Ti}$ 950) was then used to compress the pillars at room temperature under controlled displacement, with a strain rate of $2 \times 10^{-3} \mathrm{~s}^{-1}$.

At least four samples were tested for each value of diameter. In Fig. 2 we show the SEM images of the micropillars after compression for each size separately. Note the highly anisotropic, single-slip plane character of the local plastic deformation pattern in 500 and $1000 \mathrm{~nm}$ samples: the crystallographically exact slip traces are specifically indicated in Figs. 2(a) and 2(b). In $1500 \mathrm{~nm}$ crystals the plastic flow becomes more isotropic showing even locally a multislip deformation pattern.

In Fig. 3(a) we juxtaposed the stress-strain curves for Mo pillars with diameters from 500 to $1500 \mathrm{~nm}$, all showing a characteristic set of abrupt discontinuities. For the chosen pillar orientation, the slip systems with maximum Schmidt factor $S$ are (101) $\langle\overline{1} 11\rangle$ and $(011)\langle 1 \overline{1} 1\rangle$. Accordingly, after
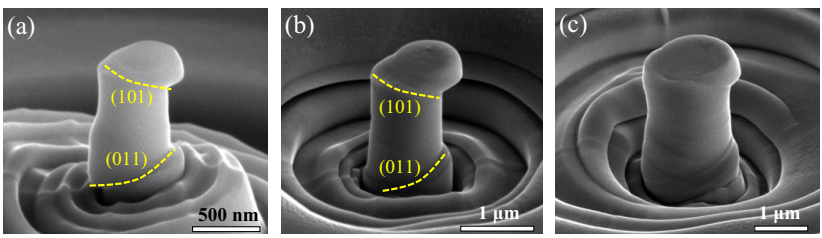

FIG. 2. SEM images of [112]-orientated micropillars after compression: (a) $500 \mathrm{~nm}$, (b) $1000 \mathrm{~nm}$, and (c) $1500 \mathrm{~nm}$. The marked slip traces in panels (a) and (b) indicate the locally single-slip nature of the plastic flow in $500 \mathrm{~nm}$ and $1000 \mathrm{~nm}$ crystals. 

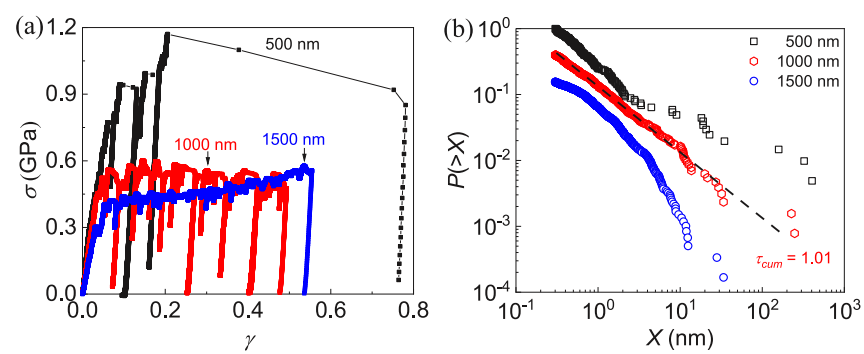

FIG. 3. Compression tests on pure Mo submicron pillars: (a) stress-strain curves (shear); (b) cumulative distribution of plastic displacements $X$ detected over the entire loading process. Here $\tau_{\text {cum }}=\tau_{\text {in }}-1$ where $\tau_{\text {in }}$ is the stress-integrated exponent.

deformation, we observed the most significant plasticity on the planes $\{110\}$.

The complex configuration of the observed jumps on the stress-strain plane can be explained by the delayed instrumental response during rapid plastic deformation; see Ref. [18] for more details. Here we only briefly mention that the mechanical loading in such experiments is performed through an autoregulation system with PID feedback. The loading device adjusts dynamically, and in the case of an avalanche, it usually does not have enough time to respond. As a result, we observe displacement jumps at an almost constant force.

The plastic displacement jumps were determined from the recorded force-displacement data using the postprocessing methodology developed in Ref. [18]. The size of dislocation avalanche was associated with the plastic displacement $X=$ $D_{e}-D_{s}+\left(F_{s}-F_{e}\right) / K_{p}$, where $D_{s}$ and $D_{e}$ are the measured displacement at the beginning of the jump and at its end, $F_{s}$ and $F_{e}$ are the corresponding values of the force, and $K_{p}$ is the independently measured stiffness of the pillar. The computed value of $X$ is expected to scale with the total distance covered by all mobile dislocations during an avalanche [45].

The cumulative probability distributions $P(X)$ are shown in Fig. 3(b) for all three sample sizes. A statistical analysis of these distributions, which involved the comparison of the pvalues and the likelihood ratios, allows us to conclude that (1) for $500 \mathrm{~nm}$ Mo micropillars the outliers observed above $X \approx$ $3 \mathrm{~nm}$ are statistically significant and indicate supercriticality; (2) for $1000 \mathrm{~nm}$ Mo micropillars, the power-law distribution is strongly supported; and (3) for $1500 \mathrm{~nm}$ Mo micropillars, both, the power law with a cutoff and the log-normal distribution are more favorable than the power-law distribution, but the log-normal distribution is more likely.

In Fig. 4(a) we illustrate the origin of the characteristic peak which is typical for the supercritical response [46]; see the green ellipse in Fig. 4(a) based on the data for four micropillars with the same diameter $500 \mathrm{~nm}$. One of these large events corresponds to the system size avalanche which is marked by the green arrow in Fig. 4(a) and is signaling the "global failure" of the sample. The midsized bursts marked in Fig. 4(b) in violet correspond to brittle events indicated in Fig. 4(a) by violet arrows. The remaining small events show at least one decade of a power-law behavior with the cumulative exponent $\tau_{\text {cum }} \approx 1.07$. The coexistence of a power-law range at small scales, with a separate peak representing system size events, is typical for spinodal criticality [47].
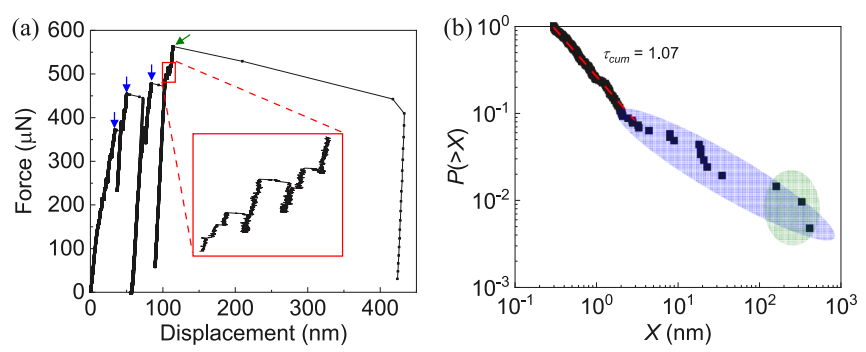

FIG. 4. Representative force-plastic displacement curve (a) and cumulative probability distribution of plastic displacements $X$ detected over the entire deformation path (b) for the Mo pillars with $500 \mathrm{~nm}$ diameter.

We note that the statistical supercriticality of nanoscale samples was not emphasized in the previous studies of the scaling in submicron crystals $[16,48,49]$. Instead, it was stressed that in almost pure crystals with negligible number of dislocations, for instance, in submicron and nanoparticles $[20,21]$, nanowires [50], or submicron pillars [22,51], the plastic deformation culminates with the formation of a system size slip band. As we show below, both phenomena have the same origin and can be ultimately linked to dislocation starvation [52].

\section{MODELING}

To simulate the observed behavior of micropillars, we use the minimal model first introduced in Ref. [36].

We can assume that the displacement field is scalar because the plastic flow of sufficiently small micropillars is mainly single-slip independently of the underlying crystal symmetry and even in the case of multislip orientation. Due to a limited number of available dislocation sources within the confined volume, the first activated slip plane dominates and prevents other slip planes from getting involved. In this situation, the usual frustration leading to hardening, can be avoided considering the absence of dislocation cross-slip and facile annihilation at a free surface. While any adequate crystal plasticity model would effectively reduce to our constrained single-slip theory in a sufficiently small system, it should, of course, allow for multislip flow to take over at larger sample sizes.

We assume that the crystal is oriented for a single slip along the only available slip direction. The crystal itself is modeled as an $N \times N$ square lattice with the mesoscopic spacing normalized to unity. The deformation of the crystal is given by the displacements of the vertices of the mesoscopic elements, $\vec{u}_{i, j}=\left(u_{i, j}^{x}, u_{i, j}^{y}\right)$, where $i, j=1,2, \ldots, N$.

In view of our single-slip assumption we allow displacements only in the horizontal direction by setting $u_{i, j}^{y} \equiv 0$. We can then introduce the notation $u_{i, j} \equiv u_{i, j}^{x}$. In the presence of a kinematic constraint the strain tensor can be reduced to two fields: a longitudinal strain, $\zeta_{i, j}=u_{i+1, j}-u_{i, j}$, which is a linear, nonorder parameter variable, and a shear strain $\xi_{i, j}=u_{i, j+1}-u_{i, j}$, which is a nonlinear, order-parametertype variable, given that plastic slip originates from multiwell nature of the lattice potential. 
We write the dimensionless energy of the system in the form [36]

$$
\Phi=\sum_{i, j} f\left(\zeta_{i, j}, \xi_{i, j}\right)
$$

where

$$
f(\zeta, \xi)=(K / 2) \zeta^{2}+f_{0}(\xi)
$$

is the energy of a single (mesoscopic) element. To account for the lattice periodicity we assume that $f_{0}(\xi)=f_{0}(\xi+n)$, where $n \in \mathbb{Z}$ is an integer-valued slip. Moreover, for analytical transparency we assume that the periodic energy density $f_{0}$ is piecewise quadratic

$$
f_{0}\left(\xi_{i, j}\right)=(1 / 2)\left[\xi_{i, j}-d_{i, j}(\xi)\right]^{2},
$$

Here the plastic slip $d$ is represented by an integer nearest to $\xi$ so that $d_{i, j}(\xi)=\left\lceil\xi_{i, j}\right\rceil$.

The obtained model depends on a single dimensionless parameter $K$ which mimics the ratios of elastic constants $\left(C_{11}-\right.$ $\left.C_{12}\right) /\left(4 C_{44}\right)$ or $C_{11} / C_{66}$. It describes the coupling between mesoscopic elements that carry different values of $\xi$. In the limits $K \rightarrow 0, \infty$ we obtain solvable $1 \mathrm{D}$ models with meanfield-type interaction [39,53]. At $K \neq 0$ the model reproduces Eshelby-type propagator and therefore captures crucial effects of long-range interactions induced by elastic compatibility; see more about this in Sec. XI. In our numerical experiments we assumed that $K=2$ which represents a typical value for metallic crystals.

The model can be reduced to a discrete automaton because the elastic problem

$$
\partial \Phi / \partial u_{i, j}=0
$$

can be solved analytically if the integer-valued field $d$ is known [36]. The associated equilibrium equations in the bulk, written in terms of the displacement field $u_{i, j}$, read

$$
\begin{aligned}
& K\left(u_{i+1, j}+u_{i-1, j}-2 u_{i, j}\right)+\left(u_{i, j+1}+u_{i, j-1}\right. \\
& \left.\quad-2 u_{i, j}\right)-\left(d_{i, j}-d_{i, j-1}\right)=0 .
\end{aligned}
$$

The whole system can be written in matrix form $\mathbf{M} u=b$, where $\mathbf{M}$ is a pentadiagonal matrix and $b$ is a vector of size $N \times N$ incorporating the boundary conditions and the field $d$. The problem then reduces to a simple matrix inversion.

We assume periodic boundary conditions in the horizontal direction $u_{1, j}=u_{N+1, j}$. The hard-device-type loading will be applied through the boundary condition in the vertical direction $u_{i, N+1}=u_{i, 1}+\gamma$, where $\gamma$ is the control parameter. Periodicity is assumed to allow for the fully explicit inversion of the matrix M. Indeed, we can then use the spectral approach based on the Fourier transform

$$
\hat{x}(\mathbf{q})=N^{-2} \sum_{a b} x_{a, b} e^{-i \mathbf{q r}}
$$

with $\mathbf{r}=(a, b)$ and $\mathbf{q}=(2 \pi k / N, 2 \pi l / N)$. In Fourier space the solution of our linear problem is straightforward and we can obtain an explicit representation for the equilibrium shear strain

$$
\hat{\xi}(\mathbf{q})=\gamma \delta(\mathbf{q})+\hat{L}(\mathbf{q}) \hat{d}(\mathbf{q})
$$

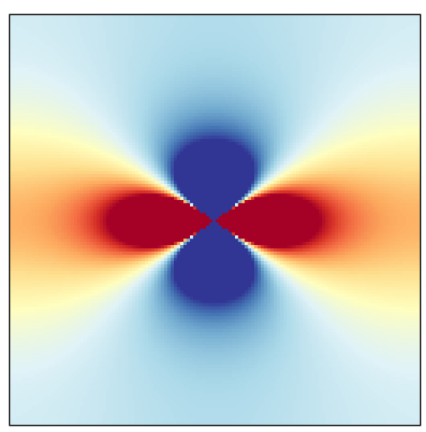

FIG. 5. The real-space representation of the dipole Fourier kernel $\hat{L}(\mathbf{q})$. Coloring: blue, negative; red, positive.

where we recall that $\gamma=\langle\xi\rangle$ is the measure of the imposed affine deformation. The sign-indefinite Eshelby-type kernel with $r^{-2}$ far-field asymptotics

$$
\hat{L}(q)=\frac{\sin ^{2}\left(q_{y} / 2\right)}{K \sin ^{2}\left(q_{x} / 2\right)+\sin ^{2}\left(q_{y} / 2\right)},
$$

is illustrated in the physical space in Fig. 5. Its dipolar structure reflects the scalar nature of our model; the more conventional quadruple structure of the stress propagator is a feature of isotropic elasticity, while here we deal with extremely anisotropic case $[54,55]$.

To illustrate dislocation nucleation in this model we show in Fig. 6 two dislocations of opposite signs forming a 2D topologically neutral "loop." The far-field asymptotics around each of the dislocations agrees with the classical continuum prediction $r^{-1}$, while inside the cores, located around the units where $d_{i+1, j}-d_{i, j} \neq 0$, the stress remains finite due to the strongly discrete nature of our theory.

Since we now know how to update the elastic fields, we can formulate the quasistatic athermal dynamics in the form of a discrete automaton for the integer-valued field $d$. We start with the unloaded $(\gamma=0)$ and dislocation-free state $\left(d_{i, j} \equiv\right.$ $0)$. We then advance the loading parameter $\gamma$ and compute (predict) the elastic field $u_{i, j}$ while keeping the field $d_{i, j}$ fixed. The knowledge of the shear strain field $\xi_{i, j}$ allows us to update (correct) the plastic strain field using the relation $d=\lceil\xi\rceil$; the update takes place when the boundary of the energy well is reached by at least one of the mesoscopic elements. Then an avalanche occurs and we use synchronous dynamics for the updates of $d_{i, j}$. We repeat the prediction-correction steps at a given $\gamma$ till the corrections stop changing the field $d_{i, j}$ and the system stabilizes in a new equilibrium state. As the
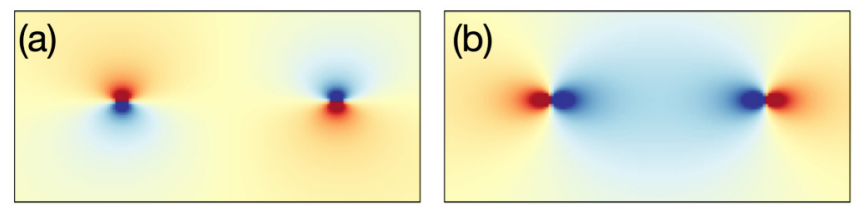

FIG. 6. Stress field produced by a pair of dislocations of different signs created using a distribution of plastic slip ( $d$ field) which vanishes everywhere except in the one element-wide 80 lattice spacings long segment. (a) Axial stress field $\sigma_{x x}$; (b) Shear stress field $\sigma_{x y}$. Coloring indicates the level of stress: blue, negative; red, positive. 
stress in this state is globally below the threshold, we can start a new search for the increment of $\delta \gamma$ that destabilizes at least one unit. As soon as such an element with $d_{i, j} \neq\lceil\xi\rceil$ is obtained we apply our relaxation protocol again, initiating another avalanche. When avalanche finishes, the variation of $\gamma$ resumes.

\section{INCOMPATIBLE DISORDER}

Given our periodic boundary conditions, we effectively consider an infinite crystal and our parameter $N$ cannot be associated with the crystal size $L$. To model the physical size effect, we would have to deal with more complex boundary conditions compatible with, say, surface dislocation nucleation and the formation of one-legged Frank-Reed loops. Without such major modifications of the model, we can study the size effect only indirectly, and we propose to use the strength of quenched disorder as a way to differentiate between submicron crystal sizes.

To justify this approach we first note that instead of $L$ one should use a dimensionless parameter, which we can always write as

$$
R=L / l,
$$

where $l$ is some appropriately chosen internal length scale and without loss of generality we can write $l \sim G b / \sigma_{t h}$, where $G$ is the shear modulus, $b$ is the Burgers vector, and $\sigma_{t h}$ is the internal stress threshold.

Following Ref. [18], we identify this threshold with the pinning (immobilization) stress. The distinctly brittle regime for semipure crystal would then correspond to small $\sigma_{t h}$ due to negligible number of obstacles ensuring that $R \ll 1$. Given that in our Mo samples the spacing of immobile dislocations $l \sim 790 \mathrm{~nm}$, brittleness of $500 \mathrm{~nm}$ pillars would be in a basic agreement with such a criterion. In the strongly ductile regime we should have $R \gg 1$, which is close to being the case for our $1500 \mathrm{~nm}$ pillars. Dislocation interaction with obstacles becomes relevant when $R \sim 1$ which is the case for our 1000 nm pillars; see Fig. 1.

The threshold $\sigma_{t h}$ naturally depends on the presence of the pinning obstacles and, in general [18], increases with the variance of quenched disorder imitating such obstacles. More specifically, the decrease of $\sigma_{t h}$ can be achieved by making the disorder more narrow which can be viewed as the way of eliminating particularly strong obstacles. Under these assumptions, instead of decreasing $L$ we can increase $l$, which should be as effective in moving from the brittle regime, where $R \ll 1$, to the ductile regime, where $R \gg 1$. In other words, instead of exploring directly the dominance of surface effects we can exploit the indirect effect that in smaller systems there are fewer strong obstacles that can serve, as dislocation nucleation sites.

It has to be mentioned, however, that our association of the variance of disorder with crystal size is exclusively targeting systems without bulk criticality, as in the case of Mo crystals. One can, in principle, manufacture small crystals with strong (dense) quenched disorder [18] or grow almost pure large crystals with very weak (sparse) quenched disorder [56]. In general, both quenched disorder and the crystal size would affect brittleness, even though to grow almost defect free crystals (without solutes, precipitates and dislocations), is almost impossible except in case of extremely small sizes (nanoparticles).

Our approach allows one to study the size effect without actually changing the size of the computational system while varying instead the strength of the quenched disorder. Here by disorder we mean first of all inclusions such as solute atoms. It can be also impurity atoms or even vacancies (or voids) resulting from the motions of dislocation jogs [57]. It will be important, however, that such disorder is elastically incompatible producing long-range effects such as in the case of local volumetric changes.

To account for such disorder in the most simple linear form, we can add to the energy density an additional term proportional to the nonorder parameter variable $\zeta$ obtaining

$$
f\left(\xi_{i, j}, \zeta_{i, j}\right)=\frac{K}{2} \zeta_{i, j}^{2}+\frac{1}{2}\left[\xi_{i, j}-d_{i, j}(\xi)\right]^{2}-h_{i, j} \zeta_{i, j}
$$

Here the random coefficients $h_{i, j}$, drawn independently in each lattice point from Gaussian distribution with variance $\delta$

$$
p(r)=\left(2 \pi \delta^{2}\right)^{-1 / 2} \exp \left[-r^{2} /\left(2 \delta^{2}\right)\right],
$$

mimic incompatible lattice prestress which acts on the order parameter variable $\xi$ only indirectly.

In the presence of quenched disorder $h$, the strain fields take the form

$$
\begin{gathered}
\hat{\xi}(\mathbf{q})=\gamma \delta(\mathbf{q})+\frac{\hat{s}_{y}^{+}(\mathbf{q})\left[\hat{s}_{x}^{-}(\mathbf{q}) \hat{h}(\mathbf{q})+\hat{s}_{y}^{-}(\mathbf{q}) \hat{d}(\mathbf{q})\right]}{2 K\left[\cos \left(q_{x}\right)-1\right]+2\left[\cos \left(q_{y}\right)-1\right]}, \\
\hat{\zeta}(\mathbf{q})=\frac{\hat{s}_{x}^{+}(\mathbf{q})\left[\hat{s}_{x}^{-}(\mathbf{q}) \hat{h}(\mathbf{q})+\hat{s}_{y}^{-}(\mathbf{q}) \hat{d}(\mathbf{q})\right]}{2 K\left[\cos \left(q_{x}\right)-1\right]+2\left[\cos \left(q_{y}\right)-1\right]},
\end{gathered}
$$

where

$$
\hat{s}_{a}^{\mp}(\mathbf{q})= \pm\left[1-\cos \left(q_{a}\right) \pm i \sin \left(q_{a}\right)\right]
$$

for $a=x, y$. We can also rewrite (12) as

$$
\hat{\xi}(\mathbf{q})=\gamma \delta(\mathbf{q})+\hat{L}(\mathbf{q}) \hat{d}(\mathbf{q})+\hat{L}_{h}(\mathbf{q}) \hat{h}(\mathbf{q}),
$$

where

$$
\hat{L}_{h}(\mathbf{q})=\frac{\sin \left(q_{x} / 2\right) \sin \left(q_{y} / 2\right)\left[\cos \left(\frac{q_{x}-q_{y}}{2}\right)-i \sin \left(\frac{q_{x}-q_{y}}{2}\right)\right]}{K \sin ^{2}\left(q_{x} / 2\right)+\sin ^{2}\left(q_{y} / 2\right)}
$$

is a distorted Eshelby propagator (8) maintaining, however, its sign indefiniteness and the decay rate $1 / r^{2}$.

\section{STRESS-STRAIN RESPONSE}

Starting with a dislocation-free crystal $(d \equiv 0)$ we now drive the system quasistatically using the athermal quasistatic protocol described above $[53,58]$. The obtained results are then averaged over 100-3000 realizations of the quenched disorder.

In Fig. 7 we illustrate the stress-strain relations $\sigma(\gamma)=$ $d \Phi / d \gamma$, where the stress was averaged over the strain interval $\sim 10^{-4}$. At each value of disorder strength $\delta$ the stress-strain curve exhibits a maximum which we conditionally identify with the yield point. Four such points are shown in Fig. 7: $A, B, C, D$. The corresponding yield strain is denoted by $\gamma_{y}$, and its dependence on disorder is shown in the inset where the 


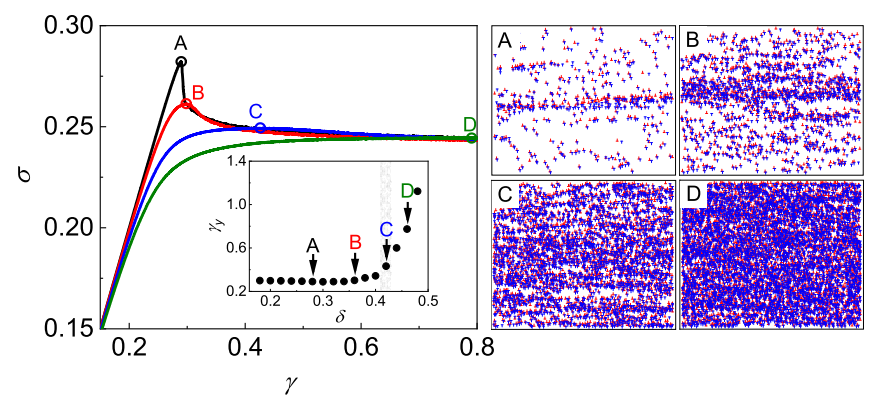

FIG. 7. The effect of disorder on the average strain-stress curves in simple shear tests. Inset shows the yield strain $\gamma_{y}$ the gray strip schematically marks the BD transition. The zoom on the "after-yield" dislocation configurations is shown in the right column where the total number of dislocations is 782 (in A), 2600 (in B), 5220 (in C), and 8862 (in D). The dislocations with positive and negative Burgers vector are marked by red and blue. Parameters: $K=2, N=1024$.

states $A, B, C, D$ are also indicated. The zoom on the "afteryield" dislocation configurations in these states is shown in the right column.

For weak disorder, $\delta \leqslant 0.3$, mimicking small, almost pure crystals, yielding is abrupt and brittle, accompanied by a macroscopic stress drop at the yield point and robust strain localization within a formation of a shear band (regime $A$ ). With increased disorder (regime $B$ ), the first-order phase transition eventually terminates at a critical point located around $\delta \sim 0.42$ (regime $C$ ); see Refs. [41,43] for similar behavior in amorphous plasticity. At even stronger disorder, $\delta \geqslant 0.5$, representing bulk samples, yielding is gradual and plasticity is ductile with slip uniformly distributed over the whole crystal (regime $D$ ).

In Fig. 8 we show the shear strain, axial stress field and shear stress patterns for samples with disorder strength marked by the letters $A, B, C, D$. In these images, the affine component of the fields was subtracted. We also present enlargement of the marked out windows.

When the disorder is weak (regime $A$ ), the shear band features a cracklike arrangement of dislocations. Outside the band, the dislocations distribution is relatively uniform, although one can trace few incipient shear prebands. As the strength of the disorder increases (regime $B$ ), the dislocation density inside the shear band diminishes and it becomes progressively broader. One can interpret this broadening as an outside propagation of the shear band boundaries. In the regime $C$, we lose a singular band which is replaced by a diffuse network of interconnected prebands which fill the whole domain. Finally, in the ductile phase, regime $D$, no coherent pattern is apparent as we see dislocation activity all over the domain. Note that the overall delocalization of the plastic flow, which we observed experimentally while increasing $L$, is recovered here as we increase $\delta$.

Note, however, that the agreement between this oversimplified theory and the experiment cannot be complete. For instance, in our physical experiments with submicron pillars, we observed repeated almost-yielding events. During such events dislocations could always annihilate on free surfaces, which was bringing the crystal into the dislocation starvation state over and over again [20]. Instead, in our computer
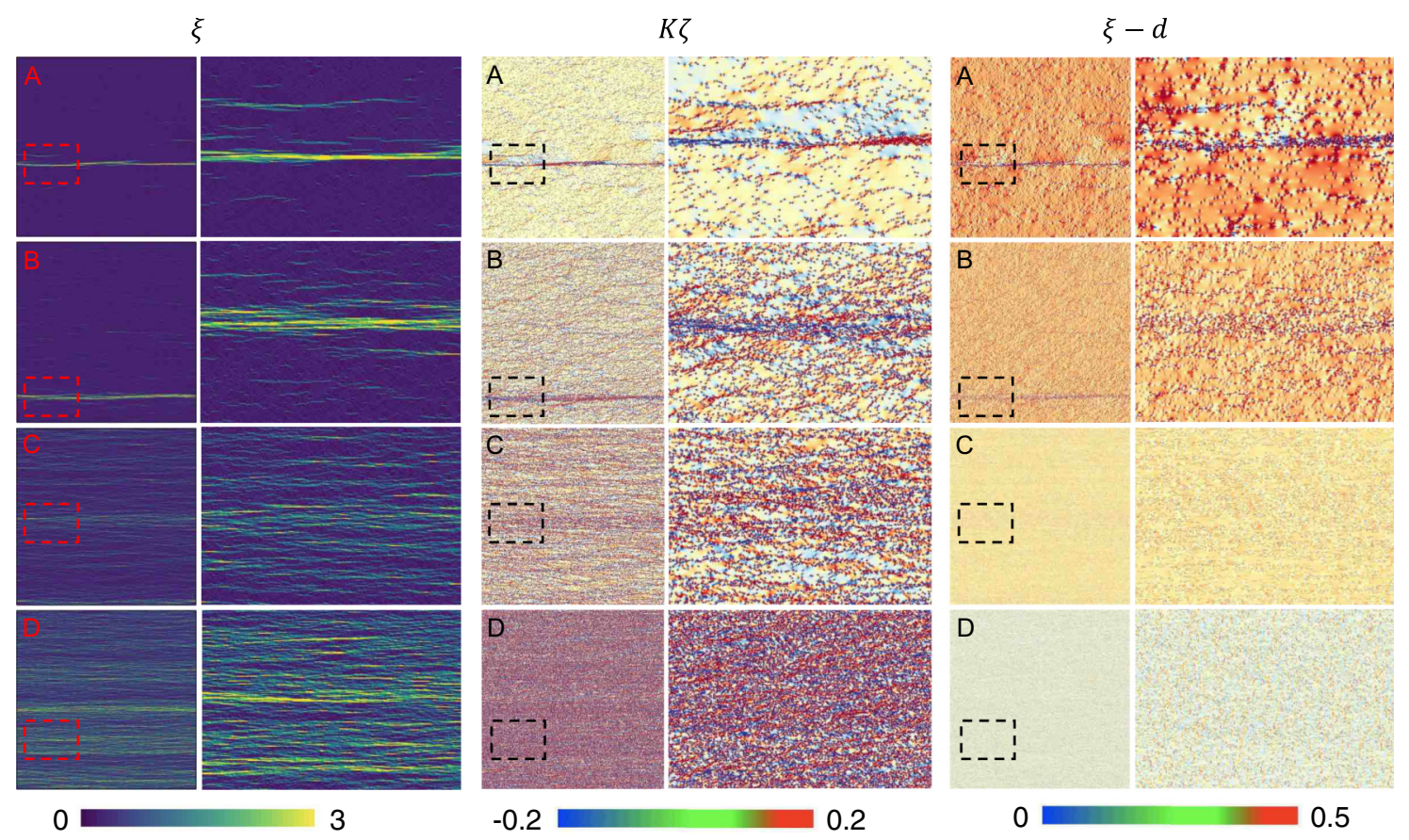

FIG. 8. After-yield spatial patterns: shear strain $\xi$, longitudinal stress $\sigma_{x x}=d f / d \zeta=K \zeta$, and shear stress $\sigma_{x y}=d f / \xi=\xi-d$ in the reference coordinates. Strength of disorder: $\delta=0.28(\mathrm{~A}), \delta=0.36(\mathrm{~B}), \delta=0.42(\mathrm{C})$ and $\delta=0.46$ (D). Zoomed views of the marked areas are shown in parallel columns. 
experiments, where we used periodic boundary conditions, such resetting did not happen because the crystal could form system-size slip bands with high dislocation density. Therefore, for each realization of disorder instead of several large bursts, we observed a single catastrophic one.

\section{SPATIAL COMPLEXITY}

As we see plastic flow proceeds through incessant mechanical destabilization and re-accomodation of dislocational microstructures. Due to the presence of long-range elastic interactions, these microstructures are not random and to reveal the nature of the implicit correlations, we performed a multifractal analysis of the field $d_{i, j}$. Originally developed in the studies of fluid turbulence, such analysis has become a powerful tool of quantifying the degree of clustering [59-62].

Multifractals were introduced to study the distribution of a scalar quantity represented by a measure (usually density, but in our case, plastic strain field). The effect to capture is that local singularities of different strengths are distributed on sets with different fractal dimensions denoted by $D_{q}$. The first of those dimensions $D_{0}$ is the fractal dimension of the geometrical support of the measure. As $q$ increases, the dimensions $D_{q}$ become more and more controlled by the most densely filled domains. An increasing difference between $D_{0}$ and $D_{q}$ with $q>0$ reveals an increasingly multiscale nature of the distribution.

To compute the dimensions $D_{q}$ in our case we need to cover the deformed lattice with a regular array of boxes of size $L_{b}$ and sum plastic strain in the $m$ th box to obtain $\mathcal{M}_{m}\left(L_{b}\right)=\sum d$, where the sum is taken over the mesoscopic units covered by a given box. Then, the density of plastic strain associated with the $m$ th box is

$$
p_{m}\left(L_{b}\right)=\frac{\mathcal{M}_{m}\left(L_{b}\right)}{\sum_{k=1}^{n\left(L_{b}\right)} \mathcal{M}_{k}\left(L_{b}\right)},
$$

where $n\left(L_{b}\right)$ is the number of boxes covering the lattice. The moments of order $q$ of this density distribution are

$$
M_{q}\left(L_{b}\right)=\sum_{m=1}^{n\left(L_{b}\right)} p_{m}^{q}\left(L_{b}\right) .
$$

If the deformation pattern is self-similar, we should observe the scaling

$$
M_{q}\left(L_{b}\right) \sim L_{b}^{(q-1) D_{q}},
$$

which defines the dimensions $D_{q}$. The singular value $D_{1}$ can be defined as the proportionality coefficient between $\sum_{m}^{n\left(L_{b}\right)} p_{m} \log \left(p_{m}\right)$ and $\log \left(L_{b}\right)$, and then $D_{q} \rightarrow D_{1}$ as $q \rightarrow 1$ $[63,64]$.

We computed the dimensions $D_{q}$ at a particular value of the loading parameter $\gamma=0.8$, where the steady flow conditions have already been achieved for all representative values of disorder $\delta$. Our results, summarized in Fig. 9, clearly show the anticipated scaling along several decades till the cutoff scale $L_{b}^{*}(\delta)$. It characterizes the spacing of the slip traces, when disorder is weak, and the spacing of the strong dislocation locks, when disorder is strong. The absence of the cutoff at $\delta=0.42$ suggests the emergence of a scale-free hierarchical microstructure.
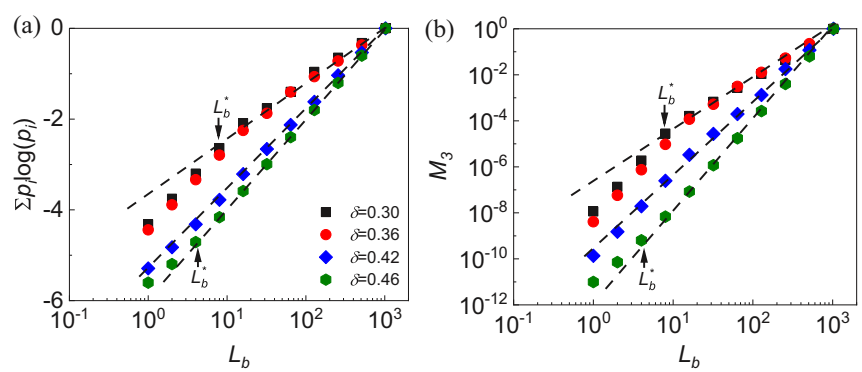

FIG. 9. Multifractal analysis of plastic strain patterns for the strain $\gamma=0.8$. (a) $\sum p_{i} \log \left(p_{i}\right)$ (" $M_{1}$ ") and (b) $M_{3}$ as a function of box size $L_{b}$.

In Fig. 10 we show the disorder dependence of the fractal dimensions $D_{q}$. For weak disorder $D_{q} \sim 1$ for all $q \geqslant 1$ which signals an extreme localization. At the $\mathrm{BD}$ transition the functions $D_{q}$ jump towards the value $\sim 2.0$, which indicates that the strain pattern becomes homogeneous. With the narrow range of disorder strengths $\delta \sim 0.42$ we can associate the emergence of a turbulence-type multifractal pattern with $D_{1}>$ $D_{2}>D_{3}>\cdots$. Regions of maximum plastic strain appear to spatially cluster on a set with fractal dimension $\sim 3 / 2$, which is also characteristic of other scale-free systems $[65,66]$.

\section{AVALANCHE STATISTICS}

Both physical and numerical experiments reveal that quasistatically driven crystals deform intermittently via avalanches reflecting destruction and rebuilding of dislocation structures. To perform a quantitative comparison of the two types of experiment we use our observation that in the automaton model the energy $E$, released during an avalanche, scales with the cumulative distance covered by the concurrently moving dislocations. The distribution $p(E)$ computed in numerical experiment will then be the analog of the experimentally measured distribution $p(X)$.

In Fig. 11 we present the three main types of cumulative distributions $P(>E)$ that emerged in our numerical experiments. Comparison with Fig. 3(b) shows that they

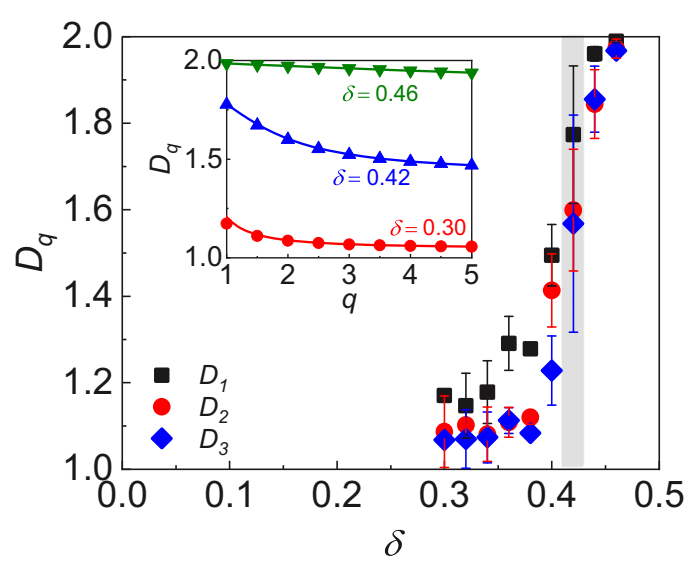

FIG. 10. Disorder dependence of the fractal measures $D_{q}$ at $\gamma=$ 0.8 ; inset reveals multifractality at critical disorder. The gray strip schematically marks the BD transition. 

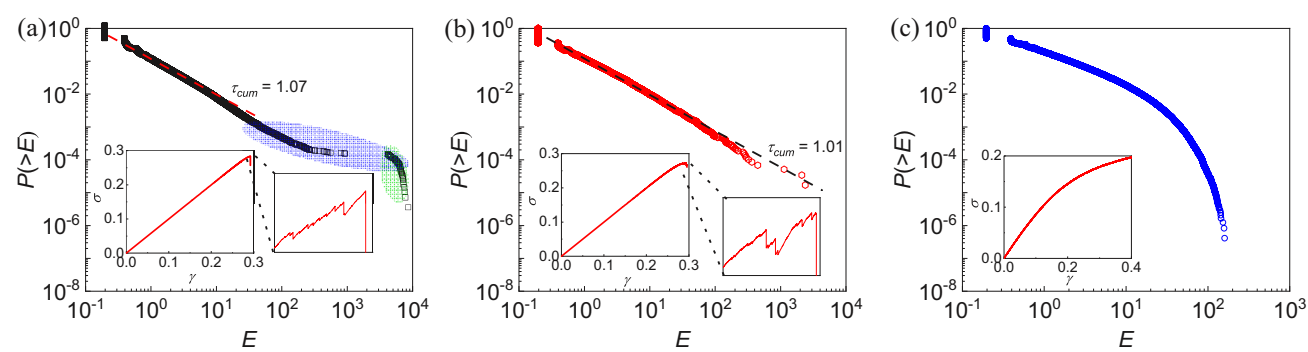

FIG. 11. Cumulative probability distributions of preyield avalanches at $\delta=0.28$ (a), $\delta=0.32$ (b), $\delta=0.7$ (c); to be compared with the data for $500 \mathrm{~nm}, 1000 \mathrm{~nm}$, and $1500 \mathrm{~nm}$ Mo samples shown in Fig. 3(b). Averaging was performed over 100 realizations of disorder; insets show the stress-strain curves for a particular realization of disorder.

reproduce all three types of the distributions $P(>X)$ recorded experimentally. Thus, at small disorder strength, our computational model captures the experimentally observed coexistence of characteristic bursts (SNAP events) with power-law distributed small avalanches observed in $500 \mathrm{~nm}$ crystals and, moreover, predicts a realistic value of the experimentally measured exponent; see Fig. 11(a). With increasing disorder the numerically obtained distribution acquires a power-law structure [see Fig. 11(b)] with the same exponent as in our data obtained from $1000 \mathrm{~nm}$ samples; see Fig. 3(b). At even larger strength of disorder we observe in our numerics the emergence of subcritical statistics; see Fig. 11(c). In this way, the model captures the observed behavior of $1500 \mathrm{~nm}$ samples dominated by largely uncorrelated POP events; see Fig. 3(b). The obtained agreement suggests that we are dealing here with very robust features of the system that are immune to structural details and indifferent to numerical values of parameters. Our results also confirm that the size effect in micropillars can be indeed successfully modeled by varying the strength of quenched disorder.

We now turn to the study of the fine details of the disorderinduced crossover phenomena that are not readily accessible in physical experiments. To this end, we approximate at each level of disorder the computed stress-resolved distributions for the released energy $E$ by the scaling relations $p(E) \sim E^{-\tau} \exp \left(-E / E_{c}\right)$ and extract the disorder-dependent exponents $\tau$ using the maximum likelihood method [67]. A clear advantage of the numerical experiment, where we could generate at least $3 \times 10^{6}$ events for each value of the exponent, is the quality of the statistics.

In Fig. 12(a) we show the obtained continuous functions $\tau(\delta)$ representing pre- and postyield exponents; the associated strain-resolved distributions are illustrated in Figs. 12(b) and 12 (c).

When the disorder is weak $(\delta \sim 0.2)$, the pre- and postyield exponents take almost the same value $\tau \sim 1$ [points $E$ and $E^{\prime}$ in Fig. 12(a)]. In such regimes, where $R<1$, homogeneously nucleated dislocations are free to self-organize under the influence of long-range elastic forces $[25,68]$ and the formation of a shear band only mildly affects global dislocation dynamics. The value of the exponent $\tau \sim 1$ presents a signature of archetypically "wild" plasticity in the sense of Ref. [29].

The exponent $\tau=1$ has previously emerged in a fully analytical mean-field theory of spin glasses where it was associated with marginal stability $[69,70]$. Based on this anal- ogy one can argue that around $\delta \sim 0.2$ our system generates sufficient self-induced disorder to undergo a transition from stable (elastic) to marginally stable (or "glassy') state whose phase space has a hierarchical (ultrametric) organization [71]. Such transition usually produces an almost gapless excitation spectrum [72] which we indeed see emerging in our system; see Sec. VIII. The analogy with spin glasses can be linked to the fact that during yielding transition, the system effectively deals with only two neighboring energy wells of the infinitely periodic local energy landscape [73].

The exponent $\tau=1$ was also obtained numerically in the studies of quasielastic regimes in structural glasses [44,74,75]. It was also found to characterize dense amorphous packings and, therefore, can be associated with the concept of jamming. In particular, the avalanche exponent $\tau=1$ is predicted by the fully analytical mean-field theory for jammed packings [70].

As we have already mentioned, the fact that the postyield avalanche distribution in these regimes is supercritical [see, for instance, our data for the $500 \mathrm{~nm}$ Mo crystals shown in Fig. 3(b) and Fig. 4(b)] is often not explicitly indicated $[16,48,49]$ even though it is well known that almost pure nano- and microcrystals always deform with a system-size dislocational avalanche [20-22,50]. The supercriticality is also suppressed by the neglect of dislocation nucleation in DDD simulations, even though the exponent $\tau \sim 1$ emerges in such models when they rely on the assumption of single-slip plasticity and neglect disorder (e.g., Ref. [32]). In fact, the
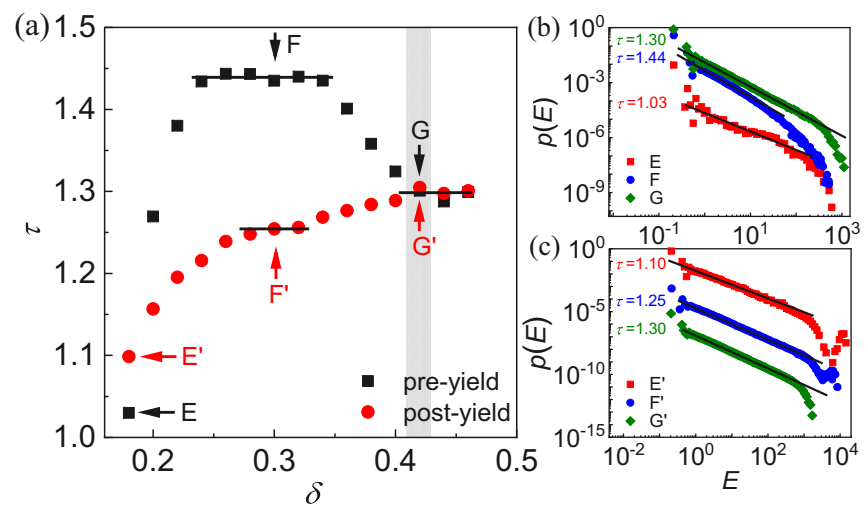

FIG. 12. (a) Disorder dependence of the stress-resolved scaling exponent $\tau$ for immediately pre- and postyield regimes; (b, c) corresponding avalanche distributions for more than 100 realizations. The gray strip schematically marks the BD transition. 


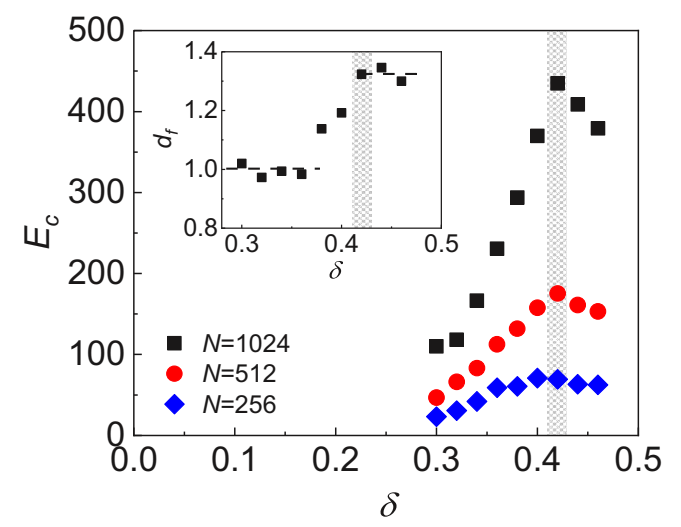

FIG. 13. The divergence of the cutoff size in the preyield regime around the critical value of disorder $\delta \sim 0.42$. Inset shows the behavior of the fractal dimension $d_{f}$; the gray strip schematically marks the BD transition.

authors of these studies have already linked such regimes with both dislocation jamming and self-induced glassiness $[33,68,76,77]$.

At the intermediate disorder range, where $0.25<\delta<$ 0.35 , we observe a gap opening between the values of pre- and postyield exponents [regimes $F$ and $F^{\prime}$ in Fig. 12(a)]. In view of the progressive rounding of the stress-strain curve near the strain controlled spinodal point, where [43] $d \sigma / d \gamma=$ $-\infty$, one can expect the preyield scaling to represent the spinodal nucleation which shows scale-free features due to the dominance of long-range elastic interactions [42,47,78,79]. In the postyield regime, the pdf's characteristic peak still indicates nucleation of system size shear bands, while the scale-free range can be linked to their spreading in the form of elastic depinning [33]. A prototypical example of a doublewell system with a long-range spinodal, where nucleation and propagation (depinning) exponents are different, is discussed in Ref. [73].

Around $\delta \sim 0.42$ the first-order phase transition terminates in a critical point representing the BD transition and the pre- and postyield exponents collapse again [regimes $G$ and $G^{\prime}$ in Fig. 12(a)]. Between $\delta \sim 0.3$ [point $F$ in Fig. 12(a)] and $\delta \sim 0.4$ [regime $G$ in Fig. 12(a)] the preyield exponent $\tau$ exhibits a characteristic disorder-induced crossover from spinodal to critical scaling discussed for the mean-field setting in Ref. [47].

In the critical BD regime at $\delta \sim 0.42$ the scaling exponent takes the value $\tau \sim 1.3$, which is close to the one observed for slip-size statistics in nanopillars (both FCC and BCC) $[8,15]$; the same value characterizes plastic yield in amorphous solids [65,80-82]. In a DDD model with quenched disorder similar value of the exponent $\tau$ was obtained in Ref. [33].

The fact that around $\delta \sim 0.42$ we encounter a critical point was already hinted upon by our finding of the multifractal structure of the plastic strain field at this strength of disorder; see Fig. 10. To provide additional evidence, we show in Fig. 13 the disorder dependence of the cutoff parameter $E_{c}$ characterizing stress-integrated preyield avalanches. It peaks at the critical value of disorder $\delta \sim 0.42$, where it diverges with system size, $E_{c} \sim N^{d_{f}}$. The fractal dimension of avalanches $d_{f}$ jumps during the BD transition from the
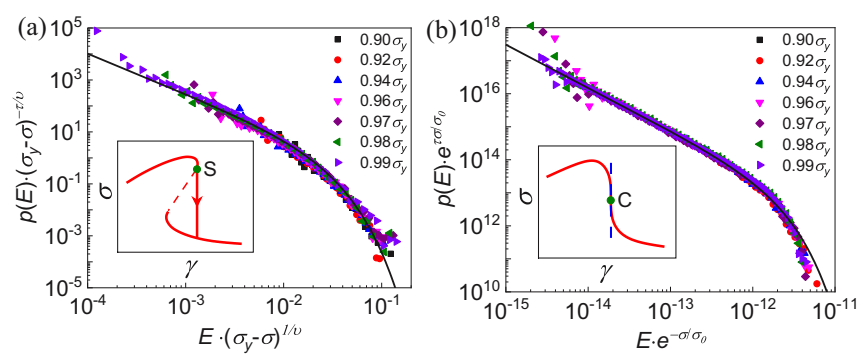

FIG. 14. Scaling collapse for the two cases: (a) $\delta=0.30$ (tuned spinodal criticality) and (b) $\delta=0.46$ (BD criticality). Insets show schematic stress-strain curves around spinodal $S$ and critical $C$ points.

value $\sim 1$, in the brittle phase $[11,32]$, to the value $\sim 1.4$ in the ductile phase. Note that the latter is close to the computed fractal dimension of the strain pattern at this level of disorder; see Sec. VI.

The BD criticality in this problem emerges within a broad range of $\delta$, which is not uncommon for systems with longrange correlations, where the presence of rare but strong spatial disorder fluctuations can divide the system into spatial regions which independently undergo the transition [83]. For a subsystem, characterized by the (average) stress-strain relation $\sigma(\gamma)$ and effectively loaded through an elastic matrix with stiffness $\mu$, the condition of criticality would be $d \sigma / d \gamma=$ $-\mu, \quad d^{2} \sigma / d \gamma^{2}=0$. The power-law distributed avalanches can be then generated in subsystems exposed to different $\mu \in$ $(0, \infty)$, even though the macroscopic critical point formally corresponds only to $\mu=\infty$.

As the strength of the disorder increases beyond $\delta \sim 0.5$, plastic hardening takes over starting at almost zero stress, and scaling is getting lost. Instead of large-scale heterogeneous avalanches, the model shows homogeneous proliferation of uncorrelated plastic activity, much like what we see in the experiments on $1500 \mathrm{~nm}$ samples; see Fig. 3(b).

Finally we show the difference in the nature of the scaling collapse of preyield data for the critical and the spinodal points. In all near-critical regimes, the stress-resolved energy distribution is of the form $p(E ; \sigma) \sim E^{-\tau} f\left[-E / E_{c}(\sigma)\right]$ and to obtain the functions $f$ and $E_{c}$, we need to replot our data using the normalized variables $E^{\prime}=E / E_{c}$ and $p^{\prime}=p(E) E_{c}^{\tau}$. We find two distinct regimes where such data collapse could be achieved; see Fig. 14.

In the interval $0.3<\delta<0.4$, Fig. 15(a) shows the validity of the scaling ansatz in the cutoff region with $E_{c}(\sigma) \sim\left(\sigma_{y}-\right.$ $\sigma)^{-1 / v}$. Here $\sigma_{y}$ is the yield stress at $\gamma_{y}$, and $v$ is a constant. This scaling suggests tuned criticality; indeed, the spinodal point is associated not only with a particular strain but also with particular stress [42,47]. We show in Fig. 15(a) that $1 / v \approx 1.6$, which is different, for instance, from the value predicted in the theory of mean-field depinning where $1 / v=$ 2.0 [84]; see Sec. XI for the relevance of this comment.

The second region of scaling collapse is around $\delta \sim$ $0.42-0.46$. Here the cutoff follows the asymptotics $E_{c}(\sigma) \sim$ $\exp \left(\sigma / \sigma_{0}\right)$, where $\sigma_{0}$ is a constant. The absence of stress tuning in this case can be explained by the fact that criticality in a strain-control ensemble makes stress poorly constrained; 

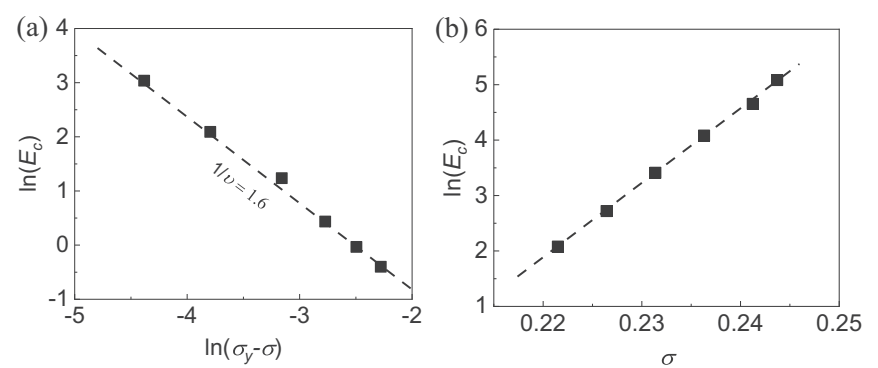

FIG. 15. Critical scaling for (a) $\delta=0.30$ (tuned spinodal criticality) and (b) $\delta=0.46$ (extended BD criticality).

see Fig. 15(b). In other words, the BD critical point at $\delta \sim$ 0.42 is localized in strain but not in stress.

\section{EXCITATION SPECTRA}

Recent advances in amorphous [80] and crystal plasticity [85] suggest that an important characterization of thresholdcontrolled dynamics comes from the density of elements with a given level of stability, also known as the excitation spectrum.

In our problem the natural stability measure is $\Delta=\bar{\xi}^{e}-\xi^{e}$ where $\xi^{e}=\xi-d(\xi)$ is the elastic strain and $\bar{\xi}^{e}=0.5$ is the stability threshold. The excitation spectrum is then the distribution $p(\Delta)$ of the local distances to a threshold above which a plastic correction takes place.

The form of the excitation spectrum in the limit $\Delta \rightarrow 0$ can be linked to the nature of intermittent fluctuations exhibited by the system $[86,87]$. Of particular interest is the pseudogap exponent $\theta(\gamma, \delta)$ entering the asymptotics $p(\Delta) \sim \Delta^{\theta}$ at $\Delta \rightarrow 0$ $[72,88]$.

We recall that in the case of classical depinning of an elastic manifold in random media, when yielding of a given site can only increase the load of other sites, it was shown that $\theta=$ 0 [89]. Another paradigmatic case is plasticity of amorphous glasses $[44,80]$ where $\theta>0$ which was linked to the fact that the elastic long range interaction kernel is sign-indefinite.

While the depinning remains one of the main paradigms of plastic yielding in crystals [12,33], it was also realized that stress transfer in crystal plasticity is sign-indefinite $[32,68,90]$, and an extensive study [85] produced a singular excitation spectrum with the exponent $\theta>0$ showing dependence on quenched disorder. The excitation spectra generated in our numerical experiments are summarized in Fig. 16.

When the quenched disorder is weak [see Fig. 16(a)], the pre- and postyield exponents $\theta$ agree. The obtained spectrum is almost gapless with very small value of $\theta>0$. This is an indication of weak criticality $[44,70,74,75,91]$ when the probability to find infinitesimal energy barrier is finite but close to zero. In such states the system is close to being elastic with dislocation microstructures characterized by high energy and low stability. Such systems are usually "marginally stable" in the sense that instabilities start to occur as soon as infinitesimal extra loading is applied, with glasses near and above jamming point as a prominent example [72]. Based only on the value of the exponent $\theta \sim 0$ it would be difficult to distinguish jamming from depinning in this case, however, since we know that $\tau \sim 1$ the jamming scenario should be clearly favored [32].

The excitation spectrum with $\theta \sim 0$ was also recorded in some mesoscopic models of amorphous plasticity [74,75] and molecular dynamic simulations of glasses [44]. As we have already mentioned in Sec. VII, this overall behavior is similar to the marginal response of mean-field systems described by the replica symmetry breaking framework and is also in agreement with what was found in simulations of three-dimensional systems of soft spheres, either at jamming or at slightly higher densities [70].

At the intermediate level of disorder $\delta \sim 0.3$ [see Fig. 16(b)], we observe the emergence of a stronger pseudogap in preyield conditions, which we interpret as a signature of spinodal criticality. Instead, the postyield regimes in this range of $\delta$ are characterized by $\theta \sim 0$. This may be explained by elastic depinning of an advancing surface separating a shear band from the rest of the crystal. Such a surface would be generically produced by a spinodal SNAP event, and its intermittent dynamics will be then controlled by sign-definite surface elasticity [73].

Around the BD critical point at $\delta \sim 0.42$ we observe a nonmonotone dependence of the exponent $\theta$ on the loading parameter with a strong maximum around the yield strain $\gamma_{y}$; see Fig. 16(c). Before the yield, we obtain $\theta \sim 0$, which can be again interpreted as a signature of dislocation jamming. The yield at this level of disorder comes with an opening of a pseudogap indicating the development of the global connectivity of the energy landscape [72,92]. The increase of the applied strain $\gamma$ beyond the critical strain, decreases the
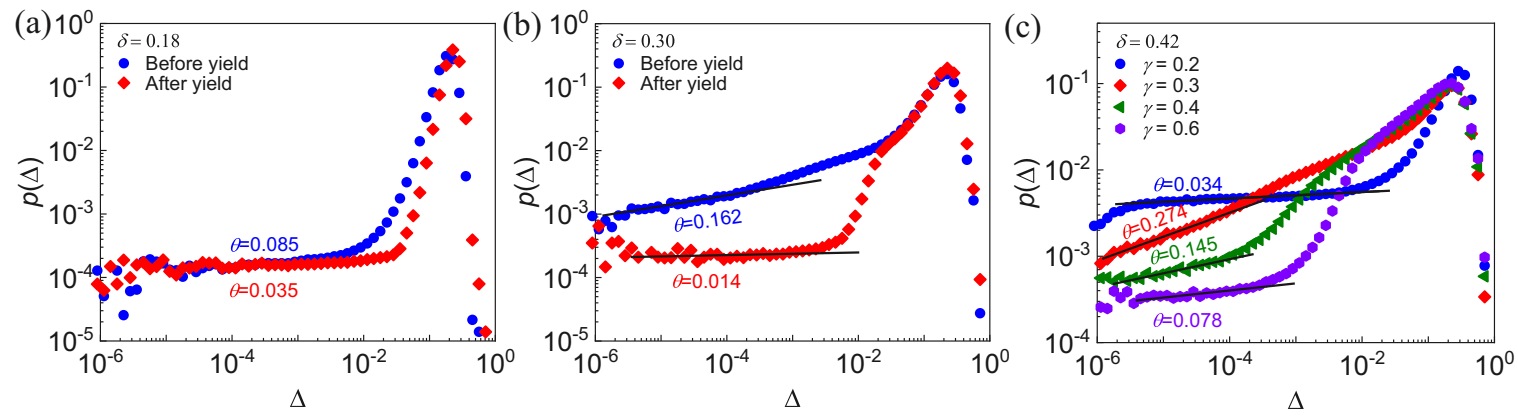

FIG. 16. Distribution of stability measures $p(\Delta$ ) at (a) $\delta=0.18$ (weak disorder), (b) $\delta=0.30$ (intermediate disorder) and (c) $\delta=0.42$ (critical disorder). In panel (c) we effectively show the function of two variables $p(\Delta ; \gamma)$. 
pseudogap exponent again, bringing it to a plateau with $\theta \sim 0$, characterizing the stationary regime [77]. We can conjecture that this as a signature of the emerging depinning scaling. Indeed, in such regimes the self-induced inhomogenity, due to dislocation entanglements, can be expected to compromise the sign-indefinite nature of the elastic kernel with long-range interactions progressively getting replaced by the largely ferromagnetic, short-range interactions.

At even stronger quenched disorder $(\delta>0.6)$ one can expect again the loss of scaling and proliferation of uncorrelated POP events.

\section{MEAN-FIELD MODEL}

A simple mean-field model can be used to rationalize at least some elements of the observed behavior in terms of macroscopic parameters. Suppose that the stress-resolved evolution of the spatially averaged density of mobile dislocations $\rho$ is described by a stochastic kinetic equation [29]

$$
\rho^{-1} d \rho / d \gamma=-c+\sqrt{2 D} \eta(\gamma),
$$

where the local shear strain $\gamma$ serves as a timelike parameter, $c \geqslant 0$ characterizes the rate of dislocation immobilization and the temperature-like parameter $D$ represents the intensity of the multiplicative mechanical noise with $\langle\eta(\gamma)\rangle=0$ and $\left\langle\eta\left(\gamma_{1}\right), \eta\left(\gamma_{2}\right)\right\rangle=\delta\left(\gamma_{1}-\gamma_{2}\right)$. Note that the lack of conventional dislocation sources in submicron crystals allows us to neglect here the Kocks-Mecking dislocation nucleation term $[29,93]$.

The stationary probability distribution in (20) is of a pure power-law form $p_{s}(\rho) \sim \rho^{-\alpha}$ with the exponent $\alpha=1+$ $c / D$. In the framework of our automaton we can interpret $\rho$ as the density of mobile dislocation during an avalanche at a given value of the loading $\gamma$. We can then write $\rho(\gamma)=$ $n(\gamma) / N^{2}$, where $n(\gamma)$ is the number of dislocations moved during an avalanche. Our numerical experiments suggest that the avalanche energy $E$ is a disorder-independent linear function of the total distance traveled by mobile dislocations during an avalanche $\bar{l}$ [see Fig. 17(b)] and that $\bar{l} \sim n$; see Fig. 17(c). Therefore $E \sim \rho$ [see Fig. 17(a)], and we can conclude that the exponent $\alpha$ in the mean-field model is the same as the exponent $\tau$ in the automaton model. The relation $\alpha=\tau$, relying on the fact that for nanocrystals the mean-free path is controlled only by the strength of quenched disorder (a proxy of the crystal size), is not applicable to bulk materials where one can instead expect that $\alpha=2 \tau-1$ [29].

For single-slip pure nanocrystals with weak disorder dislocation immobilization can be neglected, so $c / D \ll 1$, and the stochastic evolution of $\rho$ governed by (20) reduces in this case to a geometric Brownian motion with $\alpha \sim 1$. In the automaton model we observe in the low-disorder limit dislocation self-organization, governed exclusively by elastic long-range elastic interactions [32,56], and recover the same value of the exponent $\tau \sim 1$. With increasing disorder, the immobilization rate $c$ should increase leading to a higher value of $\tau$, which is in qualitative agreement with our numerical experiments.

The crossover from $D$-dominated brittle regimes $(c<D$ with stochastic term in (20) controlling dynamics) to $c$ dominated ductile regimes $(c>D$ with deterministic term in
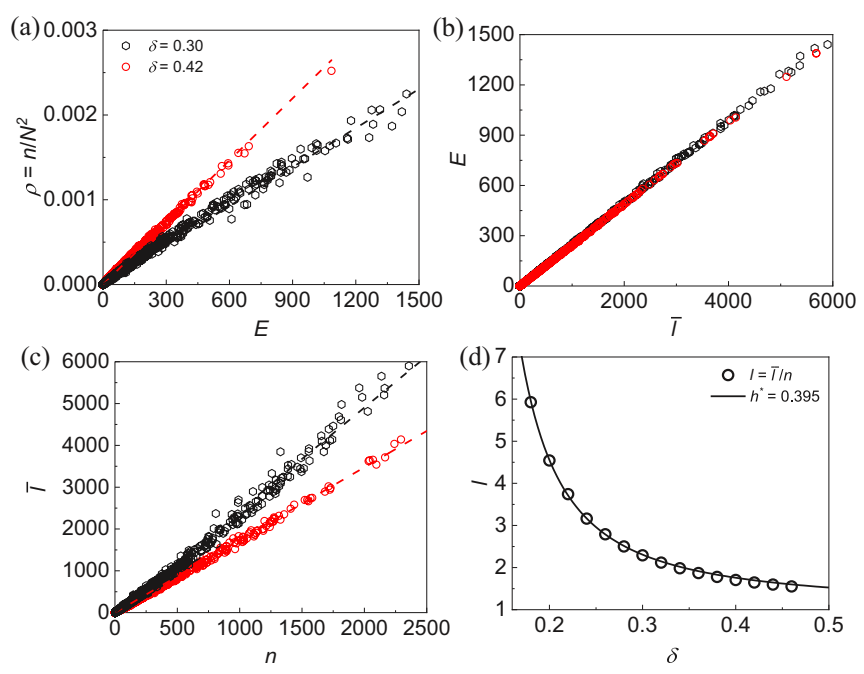

FIG. 17. (a) Relation between the density of mobile dislocations $\rho$ and avalanche energy $E$ for representative disorders $\delta=$ $0.30,0.42$; (b) dependence of $E$ on the total distance covered by dislocations during an avalanche $\bar{l}$; (c) dependence of $\bar{l}$ on the number of moving dislocations $n$; (d) internal length scale $l$ as a function of disorder strength $\delta$.

(20) controlling dynamics) can be expected where the mechanical agitation is balanced by dislocation self-locking $(c \sim$ $D$ ). Even though our oversimplified model (20) is not designed to capture the avalanche distribution in ductile regime, it is of interest to use our numerical results for reformulating the above limits in terms of crystal sizes.

Consider first the mean free path $l=\bar{l} / n$, characterizing dislocation glide before it gets immobilized. In Fig. 17(d) we show its dependence of disorder strength $\delta$ obtained from our numerical experiments. To obtain an analytical relation we can assume that only defects with the strength above some threshold $h^{*}$ participate in immobilization and that they form a regular lattice with spacing $l$. Then we can write

$$
L / l \sim\left\{1-\operatorname{erf}\left[h^{*} /(\sqrt{2} \delta)\right]\right\}^{1 / 2} .
$$

Figure 17(d) shows that this relation provides a perfect fit for the empirical curve if the stress threshold takes the value $h^{*}=0.395$. It also suggests that in the automaton model the mean free path of dislocations $l$, setting an intrinsic internal length scale, is indeed controlled by the tails of the disorder distribution.

Given that we know the relation $\tau(\delta)$ for postyield regimes from our numerical experiments [see Fig. 12(a)], and using the relation $\alpha=\tau$, we can now obtain a relation between $c / D$ and $R=L / l$; see Fig. 18. It provides the desired quantitative description of the crossover from brittle (nanocrystalline) to (ductile) microcrystalline plasticity.

Indeed, the effective temperature $D$ should depend only weakly on the system size $L$. It is defined instead by the locking strength of defects, which means that it increases with $l$. At the same time, it is clear that the rate of dislocation reactions (in particular our parameter $c$ controlling immobilization) increases with $L$ [18]. Therefore, in either very small and/or very weakly disordered samples $c<D$ and the response must be brittle. Conversely, in either bigger or more 


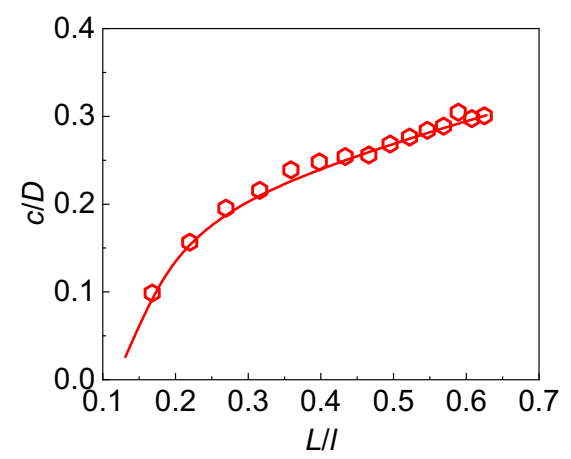

FIG. 18. Dependence of the exponent $c / D$ in the postyield regime on the dimensionless parameter $R=L / l$ for $h^{*}=0.395$ and $L=1$.

disordered samples one can expect to reach the ductile phase where $c>D$. This is what the curve shown in Fig. 18 implies.

\section{CYCLIC LOADING}

The mechanical response to monotone loading carries a memory of the initial state and in our tests, the preparation was entirely dislocation-free, as the goal was to simulate the plastic deformation of ultrasmall systems (nanoparticles and nanopillars) [24,25]. To obtain the generic response, one can prime the crystal by subjecting it to cyclic protocol. If the strain amplitude of such preloading extends beyond the yield, a crystal becomes dislocations-rich already after a first cycle, independently of the initial disorder.

The resulting self-induced disorder can be expected to quickly overtake the quenched disorder. As a result, the dimensionless parameter $R$ will increase due to the decrease of the dislocation mean free path $l$, which is now controlled by the density of the generated dislocations. This will lead to mild ductility $(R>1)$ of even submicron crystals but without strain-hardening because of the remaining single-slip arrangement of plastic flow.

The typical stress-strain curves generated by the automaton model in quasistatic cyclic loading conditions are illustrated in Fig. 19(a). Brittleness indeed disappears already after the first load reversal even for the case of relatively weak initial disorder. The decrease in yield stress is consistent with the
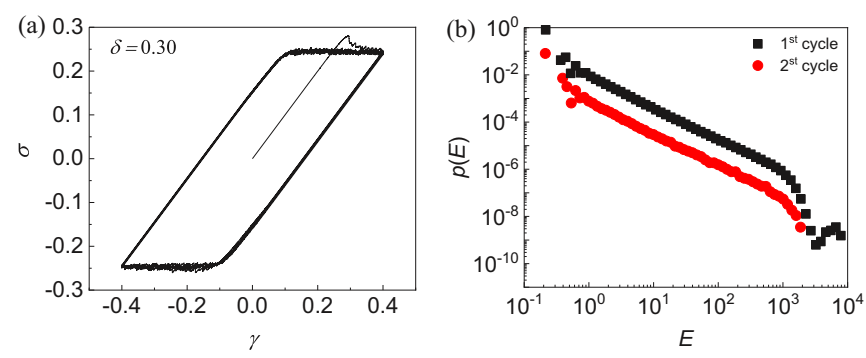

FIG. 19. (a) Strain-stress curves for the crystals showing six loading-unloading cycles; (b) Avalanche distributions of cyclically loaded crystals during the first and the second cycles; the first cycle here is understood as a monotone loading path. Here $\delta=0.30$.

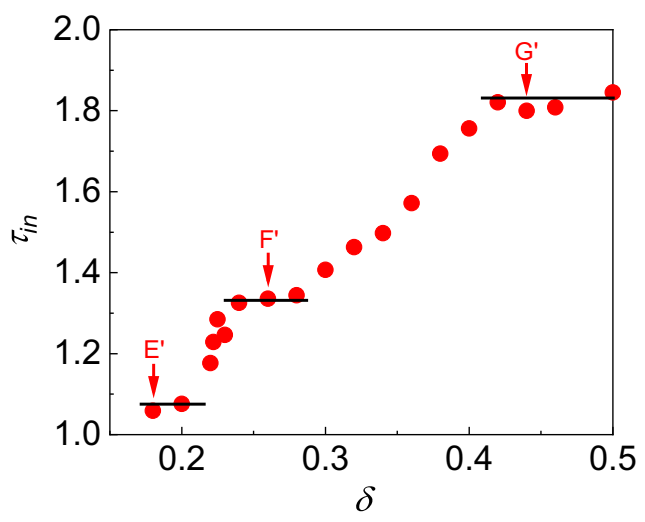

FIG. 20. Disorder dependence of the integrated exponent $\tau_{\text {int }}$ in the case of cyclic loading.

observations of softening in nanocrystals in response to the increase in the number of initial dislocations [22].

In Fig. 19(b) we show how a typical avalanche distribution changes after the first cycle for $\delta=0.30$. One can see that the originally supercritical avalanche distribution, involving characteristic system size events, is replaced by a near-critical distribution that is basically maintained by subsequent cyclic loading. A robust range of scale-free behavior with a stable exponent $\tau$ emerges after shakedown. This observation suggests that a strongly ductile regime, with $R \gg 1$ and the manifest loss of scaling, cannot be achieved by cyclic loading at least at this strength of quenched disorder.

In Fig. 20 we show the disorder dependence of the stabilized cycle-integrated exponent $\tau_{\text {in }}$ which emerges in the case of sufficiently large amplitude of cyclic loading. The $\tau_{\text {in }}(\delta)$ curve shows distinctly three characteristic plateaux corresponding to the same three main scaling regimes which we identified in Sec. VII and can conditionally label as glassy, spinodal, and critical. While the values of the exponents in Fig. 20 and in Fig. 12(a) do not match exactly due to the different nature of these exponents (stress-resolved vs. cycle-integrated) and different conditions (cyclic vs monotone loading), the main trends appear to be well maintained.

Thus, at low strength of quenched disorder (plateau around point $E^{\prime}$ ) we obtain the value of the exponent reminiscent of the one in mean-field spin glasses and suggesting that dislocations can self-organize into a marginally stable (jammed) state. At the intermediate level of disorder (plateau around point $F^{\prime}$ ) we see the scaling which we previously associated with spinodal criticality when a SNAP event produces a system size effective manifold which evolves through classical elastic depinning. Finally, at sufficiently large strength of quenched disorder (plateau around point $G^{\prime}$ ), we observe a stretched scaling range associated with the critical BD transition.

In this analysis, we have identified two major crossover regimes. The first one, from $E^{\prime}$ to $F^{\prime}$, is similar to the jamming-to-depinning transition studied numerically by DDD approach in Ref. [33]. The second one, from $F^{\prime}$ to $G^{\prime}$, is similar to the spinodal-to-critical transition studied analytically at the mean-field level in Refs. [41,43,47]. While in previous work these crossovers were associated exclusively 
with changing disorder, here we interpret them as a feature of a size effect.

Finally, we note that our observation about the disappearance of brittleness in cyclic loading can potentially be used to turn fragile nanocrystals into mildly ductile nanocrystals [4]. Given the vulnerability of brittle ultrasmall structures, the possibility to enhance ductility by purely mechanical means is of considerable interest for applications [18]. Moreover, by effectively increasing the strength of quenched disorder, such "training" can be expected to bring the crystal closer to the critical state [94].

\section{COMPATIBLE DISORDER}

In addition to disorder, characterized by the random function $h_{i, j}$, and representing prestress acting directly on the longitudinal strain variable $\zeta_{i, j}$ and indirectly on the shear strain variable $\xi_{i, j}$, one can also introduce a disorder-related prestress $g_{i, j}$ acting directly on $\xi_{i, j}$ [95]. Then the energy density takes more symmetric form [36,39]:

$$
f\left(\xi_{i, j}, \zeta_{i, j}\right)=\frac{K}{2} \zeta_{i, j}^{2}+\frac{1}{2}\left[\xi_{i, j}-d_{i, j}(\xi)\right]^{2}-h_{i, j} \zeta_{i, j}-g_{i, j} \xi_{i, j}
$$

Note that in contrast to $h_{i, j}$, the disorder $g_{i, j}$ acts on the primary order parameter variable locally as in the conventional RFIM [79]. Such "local" disorder can be viewed as resulting from lattice-compatible obstacles inhibiting or promoting plastic slip only in the narrow vicinity of a compact source of the disorder. One can think, for instance, about locked dislocation multipoles, whose long-range fields are effectively screened. "Local" disorder may also be related to lattice-scale inhomogeneities lowering or raising the Peierls stress pointwise.

Suppose that both disorder fields, $h$ and $g$, are drawn independently in each lattice cell from Gaussian distributions

$$
p_{s}(r)=\left(2 \pi \delta_{s}^{2}\right)^{-1 / 2} \exp \left[-r^{2} /\left(2 \delta_{s}^{2}\right)\right],
$$

where $s=(g, h)$. In the previous sections we used the special notation $\delta \equiv \delta_{h}$ but here, to distinguish the two, we'll keep the notation $\delta_{h}$.

The specificity of the disorder $g_{i, j}$, representing essentially a residual plastic strain, is that it can be simply combined in the energy density with the actual plastic strain $d_{i, j}$. For instance, to account for $g$ in the Fourier representation of the elastic solution, it is sufficient to replace the field $\hat{d}(\mathbf{q})$ by the sum $\hat{g}(\mathbf{q})+\hat{d}(\mathbf{q})$. We can then write

$$
\hat{\xi}(\mathbf{q})=\gamma \delta(\mathbf{q})+\hat{L}(\mathbf{q})[\hat{d}(\mathbf{q})+\hat{g}(\mathbf{q})]+\hat{L}_{h}(\mathbf{q}) \hat{h}(\mathbf{q}),
$$

where the kernels $\hat{L}_{h}(\mathbf{q})$ and $\hat{L}_{h}(\mathbf{q})$ were introduced in (8) and (16), respectively.

To perform a direct comparison of the two types of disorder we need to assess the action of the fields $g_{i, j}$ and $h_{i, j}$ on the same strain variable. A natural way to do this is to eliminate the linear nonorder parameter $\zeta_{i, j}$ adiabatically and to evaluate the role of disorder $h_{i, j}$ in the "condensed" model containing only one variable $\xi_{i, j}$. The crucial observation is that the strain variables $\xi_{i, j}$ and $\zeta_{i, j}$ are not independent even though they are not coupled explicitly in the energy density. The
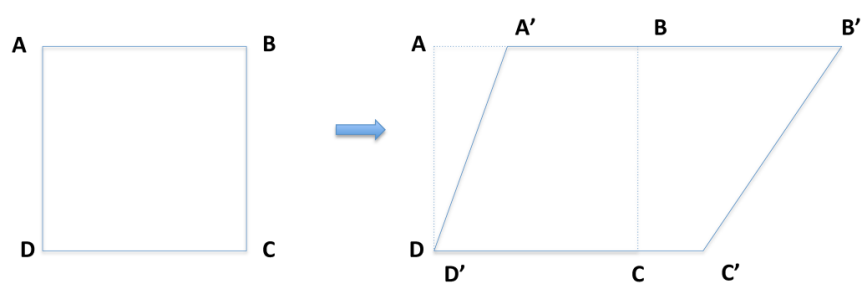

FIG. 21. Generic deformation of a mesoscopic square element.

implicit coupling can be revealed if we recall the constraint of geometric compatibility [96].

From now on, it will be convenient to deal directly with physical variables rather than their Fourier transforms. Since our displacement field is scalar, the generic deformation of an element is highly anisotropic; see Fig. 21. The geometrical meaning of the two strain variables $\xi_{i, j}$ and $\zeta_{i, j}$ becomes apparent from the identification: $D^{\prime} C^{\prime}=1+\zeta_{i, j}, A^{\prime} B^{\prime}=1+$ $\zeta_{i, j+1}, A A^{\prime}=\xi_{i, j}$, and $B B^{\prime}-C C^{\prime}=\xi_{i+1, j}$, where $A B C D$ is the square lattice element before the deformation and $A^{\prime} B^{\prime} C^{\prime} D^{\prime}$ is its image after the deformation; see Fig. 21.

It is straightforward to see that $A A^{\prime}+A^{\prime} B^{\prime}=1+B B^{\prime}$, and since $D^{\prime} C^{\prime}-C C^{\prime}=1$ we obtain in terms of $\xi_{i, j}$ and $\zeta_{i, j}$ [94]

$$
\xi_{i, j}-\xi_{i+1, j}=\zeta_{i, j}-\zeta_{i, j+1}
$$

Equation (25) is a discrete analog of the classical SaintVenant compatibility relations in continuum linear elasticity; see Ref. [97] for the general analysis. It provides a constraint on the variables $\xi_{i, j}$ and $\zeta_{i, j}$ which is inherently nonlocal. If we now complement the condition (25) with our (single) mechanical equilibrium condition (4), we obtain a closed system representing in our case the classical Beltrami-Michell equations of classical elasticity [98].

To simplify notations we'll be using lexicographic order of the elements expressing the Cartesian coordinates $(i, j)$ in terms of a single label $p=i+(j-1) N$ that takes values $p=$ $1,2, \ldots N \times N$. With these notations, a second-order tensor can be represented as a vector and a fourth-order tensor takes the form of a matrix.

Consider for simplicity an externally unloaded body. Using the lexicographic notations we can write the equilibrium equation $\partial \Phi / \partial u=0$, in the form $K \zeta=h+\mathbf{A}[\xi-(d+g)]$, where $\mathbf{A}$ is a standard fourth-order tensor with constant entries. Substituting the expression for $\zeta$ into the compatibility equations (25) we obtain $h+\mathbf{A}[\xi-(d+g)]=K \mathbf{B} \xi$, where $\mathbf{B}$ is another standard forth order tensor; the explicit expressions for the tensors $\mathbf{A}$ and $\mathbf{B}$ can be found in Ref. [94].

Using the obtained relations we can write the explicit representation of elastic solution in the form

$$
\xi=\left(\mathbf{I}-K \mathbf{A}^{-1} \mathbf{B}\right)^{-1}\left[(d+g)-\mathbf{A}^{-1} h\right] .
$$

We stress that the "local" disorder $g$ enters (26) as a quenched analog of a compatible plastic deformation. Instead the incompatible disorder $h$ enters the solution nonlocally in the sense that a residual stress $h$ placed in the element $\{k, l\}$ affects the actual elastic strain field $\xi$ in every other element $\{i, j\}$.

Note also that since plastic slip develops to minimize elastic energy, it effectively acts to bring the expression in square brackets in (26) closer to zero. It can then compensate 


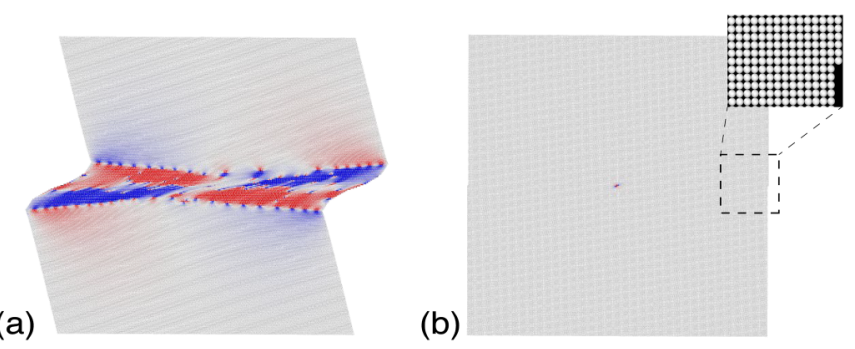

FIG. 22. Comparison of the displacement fields resulting from pointwise "nonlocal" and "local" disorders: (a) $g_{i, j} \equiv 0$ and $h_{i, j}=$ $0.5 \delta_{i, 0} \delta_{j, 0}$; (b) $g_{i, j}=0.5 \delta_{i, 0} \delta_{j, 0}$ and $h_{i, j} \equiv 0$. The "nonlocal" disorder produces a shear band with many dislocations. The "local" disorder produces a single slip due to nucleation and subsequent annihilation of a pair of dislocations. Here $K=2, N=256$; colors reflect the level of longitudinal strain.

a compact source of the disorder $g$ by yielding locally, at the location of such source. Instead, a compact source of the disorder $h$ can be compensated only by a broadly distributed plastic slip. To illustrate this point, we compare in Fig. 22 the responses of a loaded crystal with either "nonlocal" disorder $h$ or "local" disorder $g$ present in the form of a point source.

Specifically, we consider the disorder fields $g_{i, j}=p \delta_{i, 0} \delta_{j, 0}$ and $h_{i, j}=q \delta_{i, 0} \delta_{j, 0}$, where $\delta_{i, j}$ is the Kronecker delta, and choose the amplitudes $p=q$ to ensure that when only one of these fields is present at $\gamma=0$ no plasticity occurs. Then, in each of these two cases, we find the smallest increment $\delta \gamma$ initiating a slip in at least one element.

If $p=0$ and $q=0.5$ (minimal "nonlocal" disorder), the avalanche resulting from such loading is dramatic with many dislocations forming collectively and the system developing a macroscopic shear band with complex internal structure; see Fig. 22(a). If $p=0.5$ and $q=0$ (minimal "local" disorder), two dislocations of opposite sign nucleate at the source of the disorder and move apart to finally annihilate at the boundaries where we impose periodic boundary conditions. Therefore the response remains contained and reduces to the formation of a microscopic slip at the scale of a single element; see Fig. 22(b).

The observed nonlocal (global) accommodation of the disorder $h$ is possible only when the system is sufficiently homogeneous. In the presence of a substantial "local" disorder $g$, the ability of the system to generate such global response may be compromised. At sufficient strength of "local" disorder $\delta_{g}$ the coherent accommodation of "nonlocal" disorder $\delta_{h}$ will become impossible, and as we argue below, this can change the avalanche scaling in a fundamental way.

To avoid any dependence on the initial preparation of the sample, we have chosen to present the interplay between the two types of disorder, "local" and "nonlocal" in the setting of cyclic loading. Our numerical experiments, summarized in Fig. 23(a), show that when a weak "local" disorder $\delta_{g}=0.3$ is combined with a weak "nonlocal" disorder $\delta_{h}=0.3$, the overall mechanical response is ductile. The initial softening behavior, observed in crystals with $\delta_{g}=0$, is replaced by the more conventional hardening behavior. At large strains the stress response shows a robust yielding plateau independently of the configuration of disorder. The overall response is remi-
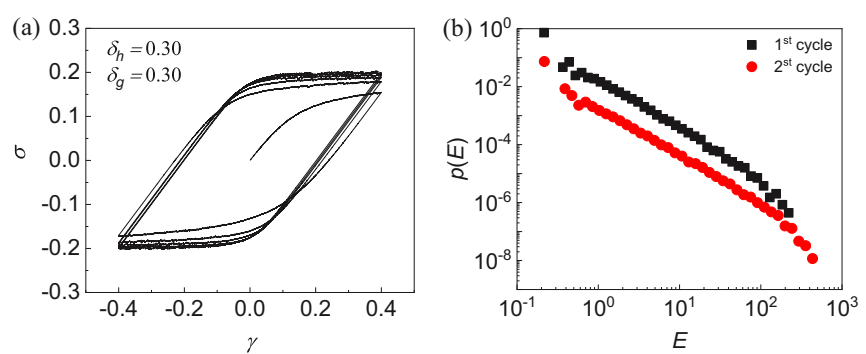

FIG. 23. (a) Strain-stress curves for the crystals subjected to six loading unloading cycles. (b) Avalanche distributions of cyclically loaded crystals for the first and the second cycles; the first cycle is understood as the monotone loading path. Here $\delta_{h}=\delta=0.30, \delta_{g}=$ 0.30 .

niscent of the classical strain-hardening behavior exhibited by bulk FCC and BCC materials [99].

From Fig. 23(b) we see that even a weak "local" disorder is sufficient to suppress supercriticality and to completely eliminate system-size events. This observation agrees with the idea that such disorder generates local inhomogeneities which inhibit global response. However, the increase of the cutoff size in the second cycle suggests that a correlated behavior, reminiscent of disorder-induced self-organization towards classical criticality in RFIM model [40,47], can still take place.

In Fig. 24 we show how the different configurations of "local" and "nonlocal" disorder strengths affect the cycleaveraged (integrated) scaling exponents $\tau_{i n}$. When the "local" disorder is weak, we recover the after-yield behavior studied in Sec. X. At stronger "local" disorder, the dependence of the exponent $\tau_{\text {in }}$ on the "nonlocal" disorder progressively diminishes. Beyond $\delta_{g} \sim 0.25$ it completely disappears, and the exponent stabilizes around the value $\tau_{\text {in }} \sim 3 / 2$. Given that the statistics is mostly acquired during hardening-free yield (see Fig. 23), one can expect the stress resolved value of the exponent $\tau$ to be similar to the aggregate value $\tau_{\text {in }}$ [100]. In this case the obtained exponent value suggests mean-field criticality $[40,47]$. In other words, the abundance of "local" disorder apparently trivializes the scaling picture, erasing the

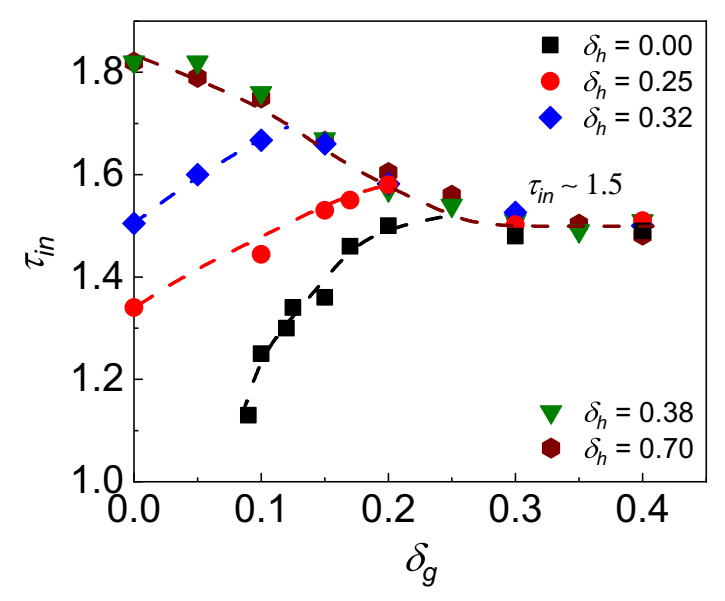

FIG. 24. Effect of the "local" disorder $\delta_{g}$ on the (integrated) scaling exponent $\tau_{\text {in }}$ for the case of cyclic loading. 
nonuniversality and promoting a universal response of the athermally driven infinite dimensional RFIM, also dominating the response of amorphous solids [41,101-103].

\section{CONCLUSIONS}

To address the fundamental question why the dislocation avalanches in submicron crystals of both face-centered cubic (fcc) and body-centered cubic (bcc) metals exhibit "wild" scaling, while the associated bulk crystals are "mild," we conducted a range of numerical experiments using a minimal integer-automaton model of crystal plasticity.

Our approach to the study of the size effect is based on the assumption that the dominance of surface-induced dislocation activity in submicron crystals can be modeled by the scarcity of conventional bulk dislocation sources. To justify this assumption, we compared the effects of extreme miniaturization in our physical experiments on Mo micropillars with the behavior of the numerical model as we progressively diminished the strength of quenched disorder. In both cases, we observed the same second-order BD transition, which provides the basic explanation for the ultimate shift from wildness to mildness in the fluctuational response with nonuniversality ultimately emerging as a size effect.

The detailed transition from largely brittle to mostly ductile behavior was conceptualized as a complex three-stage crossover. The individual transitions are from spin-glass-type marginality, characteristic of very small, almost disorder free crystals, through spinodal or depinning criticality at intermediate sizes (moderate disorder level), to the classical BD criticality in larger, highly disordered crystals. In general, this scenario shows some similarity with the one observed in amorphous plasticity $[41,43]$, however, the nuanced picture in crystal plasticity appears to be more intricate.

In addition to monotone loading, we also considered largeamplitude oscillatory shear loading protocols. We observed that brittleness disappears after cyclic loading, which suggests that nominally brittle submicron crystals can be trained to become ductile. However, the basic crossover structure, including the presence of three distinct universality classes, was shown not to be affected by the type of loading.

The nonuniversality of the scaling behavior progressively weakens as we complement the generic incompatible disorder, interacting with plasticity nonlocally, by the more special compatible disorder, which affects the plastic slip locally as in RFIM model. Our numerical experiments suggest that the increase in the strength of the "local" disorder eventually restores universality bringing the system into the mean-fieldtype criticality class characteristic of amorphous solids.

Our schematic scalar model would have to be extended to the full tensorial theory based on nonlinear elasticity to obtain a more detailed description of crystal plasticity. This extension will allow one to distinguish between different crystallographic classes and different configurations of dislocation cores. The resulting model should be able to reproduce dislocation walls and generate cell structures with realistic size distribution. A 3D theory of this type can also capture dislocation cross-slip and address hardening behavior. To study surface effects directly, one would need to come up with the boundary conditions allowing for dislocation nucleation on the surface. A path in this general direction has been recently sketched in Ref. [104].

\section{ACKNOWLEDGMENTS}

We thank N. Gorbushin, M. Mungan, F. Perez Reche, and D. Vandembroucq for helpful discussions and Jinyu Zhang for the characterization of dislocation structure. This work was supported by French-Chinese ANR-NSFC grant (ANR-19-CE08-0010-01 and 51761135031). P.Z. acknowledges additional support from the China Scholarship Council and China Postdoctoral Science Foundation (grant 2019M653595).
[1] W.-Z. Han, L. Huang, S. Ogata, H. Kimizuka, Z.-C. Yang, C. Weinberger, Q.-J. Li, B.-Y. Liu, X.-X. Zhang, J. Li, E. Ma, and Z.-W. Shan, Adv. Mater. 27, 3385 (2015).

[2] R. Maßß, L. Meza, B. Gan, S. Tin, and J. R. Greer, Small 8, 1869 (2012).

[3] D. Mordehai, S.-W. Lee, B. Backes, D. J. Srolovitz, W. D. Nix, and E. Rabkin, Acta Mater. 59, 5202 (2011).

[4] S. Papanikolaou, Y. Cui, and N. Ghoniem, Modell. Simul. Mater. Sci. Eng. 26, 013001 (2017).

[5] R. Maaß and P. M. Derlet, Acta Mater. 143, 338 (2018).

[6] M. D. Uchic, D. M. Dimiduk, J. N. Florando, and W. D. Nix, Science 305, 986 (2004).

[7] J. R. Greer, W. C. Oliver, and W. D. Nix, Acta Mater. 53, 1821 (2005).

[8] D. M. Dimiduk, C. Woodward, R. Lesar, and M. D. Uchic, Science 312, 1188 (2006).

[9] M. Zaiser, Adv. Phys. 55, 185 (2006).

[10] M. D. Uchic, P. A. Shade, and D. M. Dimiduk, Annu. Rev. Mater. Res. 39, 361 (2009).
[11] F. F. Csikor, C. Motz, D. Weygand, M. Zaiser, and S. Zapperi, Science 318, 251 (2007).

[12] N. Friedman, A. T. Jennings, G. Tsekenis, J.-Y. Kim, M. Tao, J. T. Uhl, J. R. Greer, and K. A. Dahmen, Phys. Rev. Lett. 109, 095507 (2012).

[13] R. Maass, M. Wraith, J. T. Uhl, J. R. Greer, and K. A. Dahmen, Phys. Rev. E 91, 042403 (2015).

[14] K. S. Ng and A. H. W. Ngan, Acta Mater. 56, 1712 (2008).

[15] S. Brinckmann, J.-Y. Kim, and J. R. Greer, Phys. Rev. Lett. 100, 155502 (2008).

[16] M. Zaiser, J. Schwerdtfeger, A. Schneider, C. Frick, B. G. Clark, P. Gruber, and E. Arzt, Philos. Mag. 88, 3861 (2008).

[17] S. Papanikolaou, D. M. Dimiduk, W. Choi, J. P. Sethna, M. D. Uchic, C. F. Woodward, and S. Zapperi, Nature (London) 490, 517 (2012).

[18] P. Zhang, O. U. Salman, J.-Y. Zhang, G. Liu, J. Weiss, L. Truskinovsky, and J. Sun, Acta Mater. 128, 351 (2017).

[19] Y. Cui, G. Po, and N. Ghoniem, Phys. Rev. B 95, 064103 (2017). 
[20] Z.-J. Wang, Z.-W. Shan, J. Li, J. Sun, and E. Ma, Acta Mater. 60, 1368 (2012).

[21] D. Chrobak, N. Tymiak, A. Beaber, O. Ugurlu, W. W. Gerberich, and R. Nowak, Nat. Nanotechnol. 6, 480 (2011).

[22] H. Bei, S. Shim, G. M. Pharr, and E. P. George, Acta Materialia 56, 4762 (2008).

[23] K. B. Broberg, Cracks and Fracture (Elsevier, San Diego, USA, 1999).

[24] A. Sharma, J. Hickman, N. Gazit, E. Rabkin, and Y. Mishin, Nat. Commun. 9, 4102 (2018).

[25] D. Mordehai, O. David, and R. Kositski, Adv. Mater. 30, 1706710 (2018).

[26] A. S. Argon, Philos. Mag. 93, 3795 (2013).

[27] A. A. Benzerga, J. Mech. Phys. Solids 57, 1459 (2009).

[28] C. Motz, D. Weygand, J. Senger, and P. Gumbsch, Acta Mater. 57, 1744 (2009).

[29] J. Weiss, W. B. Rhouma, T. Richeton, S. Dechanel, F. Louchet, and L. Truskinovsky, Phys. Rev. Lett. 114, 105504 (2015).

[30] T. Niiyama and T. Shimokawa, Phys. Rev. E: Stat. Nonlin. Soft Matter Phys. 91, 022401 (2015).

[31] M. C. Miguel, A. Vespignani, S. Zapperi, J. Weiss, and J. R. Grasso, Nature (London) 410, 667 (2001).

[32] P. D. Ispánovity, L. Laurson, M. Zaiser, I. Groma, S. Zapperi, and M. J. Alava, Phys. Rev. Lett. 112, 235501 (2014).

[33] M. Ovaska, L. Laurson, and M. J. Alava, Sci. Rep. 5, 10580 (2015).

[34] P. Y. Chan, G. Tsekenis, J. Dantzig, K. A. Dahmen, and N. Goldenfeld, Phys. Rev. Lett. 105, 015502 (2010).

[35] M. Zaiser and N. Nikitas, J. Stat. Mech. (2007) P04013.

[36] O. U. Salman and L. Truskinovsky, Phys. Rev. Lett. 106, 175503 (2011).

[37] G. Sparks and R. Maaß, Phys. Rev. Mater. 2, 120601 (2018).

[38] H. Song, D. Dimiduk, and S. Papanikolaou, Phys. Rev. Lett. 122, 178001 (2019).

[39] O. U. Salman and L. Truskinovsky, Int. J. Eng. Sci. 59, 219 (2012).

[40] K. Dahmen and J. P. Sethna, Phys. Rev. B 53, 14872 (1996).

[41] M. Ozawa, L. Berthier, G. Biroli, A. Rosso, and G. Tarjus, Proc. Natl. Acad. Sci. USA 115, 6656 (2018).

[42] I. Procaccia, C. Rainone, and M. Singh, Phys Rev E 96, 032907 (2017).

[43] M. Popović, T. W. J. de Geus, and M. Wyart, Phys. Rev. E 98, 040901(R) (2018).

[44] B. Shang, P. Guan, and J.-L. Barrat, Proc. Natl. Acad. Sci. USA 117, 86 (2020).

[45] R. Maaß, P. M. Derlet, and J. R. Greer, Scr. Mater. 69, 586 (2013).

[46] D. Sornette and G. Ouillon, Eur. Phys. J. Spec. Top. 205, 1 (2012).

[47] H. Borja da Rocha and L. Truskinovsky, Phys. Rev. Lett. 124, 015501 (2020).

[48] Y. Cui, G. Po, P. Srivastava, K. Jiang, V. Gupta, and N. Ghoniem, Int. J. Plast. 124, 117 (2020).

[49] G. Sparks, Y. Cui, G. Po, Q. Rizzardi, J. Marian, and R. Maaß, Phys. Rev. Mater. 3, 080601 (2019).

[50] Y. Lu, J. Song, J. Y. Huang, and J. Lou, Nano Res. 4, 1261 (2011).

[51] I. Issa, J. Amodeo, J. Réthoré, L. Joly-Pottuz, C. Esnouf, J. Morthomas, M. Perez, J. Chevalier, and K. Masenelli-Varlot, Acta Mater. 86, 295 (2015).
[52] J. R. Greer and W. D. Nix, Phys. Rev. B Condens. Matter 73, 245410 (2006).

[53] G. Puglisi and L. Truskinovsky, J. Mech. Phys. Solids 53, 655 (2005).

[54] G. Picard, A. Ajdari, F. Lequeux, and L. Bocquet, Eur. Phys. J. E 15, 371 (2004).

[55] B. Tyukodi, S. Patinet, S. Roux, and D. Vandembroucq, Phys. Rev. E 93, 063005 (2016).

[56] J. Weiss, Philos. Trans. A: Math. Phys. Eng. Sci. 377, 20180260 (2019).

[57] A. Deschamps, G. Fribourg, Y. Brechet, J. L. Chemin, and C. Hutchinson, Acta Mater. 60, 1905 (2012).

[58] C. E. Maloney and A. Lemaître, Phys. Rev. E: Stat. Nonlin. Soft Matter Phys. 74, 016118 (2006).

[59] G. Paladin and A. Vulpiani, Phys. Rep. 156, 147 (1987).

[60] B. B. Mandelbrot, Fractals in Geophysics (Springer, Birkhauser, Basel, 1989), pp. 5-42.

[61] C. Meneveau and K. Sreenivasan, J. Fluid Mech. 224, 429 (1991).

[62] R. Lopes and N. Betrouni, Med. Image Anal. 13, 634 (2009).

[63] J. Weiss, Eng. Fract. Mech. 68, 1975 (2001).

[64] M. A. Lebyodkin and T. A. Lebedkina, Phys. Rev. E 73, 036114 (2006).

[65] K. M. Salerno and M. O. Robbins, Phys. Rev. E 88, 062206 (2013).

[66] F. Gimbert, D. Amitrano, and J. Weiss, EPL 104, 46001 (2013).

[67] A. Clauset, C. R. Shalizi, and M. E. Newman, SIAM Rev. 51, 661 (2009).

[68] A. Lehtinen, G. Costantini, M. J. Alava, S. Zapperi, and L. Laurson, Phys. Rev. B 94, 064101 (2016).

[69] F. Pázmándi, G. Zaránd, and G. T. Zimányi, Phys. Rev. Lett. 83, 1034 (1999).

[70] S. Franz and S. Spigler, Phys. Rev. E 95, 022139 (2017).

[71] L. Berthier, G. Biroli, P. Charbonneau, E. I. Corwin, S. Franz, and F. Zamponi, J. Chem. Phys. 151, 010901 (2019).

[72] M. Müller and M. Wyart, Ann. Rev. Condens. Matter Phys. 6, 177 (2015).

[73] F.-J. Pérez-Reche, L. Truskinovsky, and G. Zanzotto, Phys. Rev. Lett. 101, 27 (2008).

[74] B. Tyukodi, D. Vandembroucq, and C. E. Maloney, Phys. Rev. E 100, 043003 (2019).

[75] E. E. Ferrero and E. A. Jagla, Soft Matter 15, 9041 (2019).

[76] X. Zhang, X.-C. Zhang, Q. Li, and F.-L. Shang, Chin. Phys. Lett. 33, 106401 (2016).

[77] C. Ruscher and J. Rottler, arXiv:1908.01081 (2019).

[78] C. D. Ferguson, W. Klein, and J. B. Rundle, Phys. Rev. E 60, 1359 (1999).

[79] S. K. Nandi, G. Biroli, and G. Tarjus, Phys. Rev. Lett. 116, 145701 (2016).

[80] J. Lin, E. Lerner, A. Rosso, and M. Wyart, Proc. Natl. Acad. Sci. USA 111, 14382 (2014).

[81] C. Liu, E. E. Ferrero, F. Puosi, J.-L. Barrat, and K. Martens, Phys. Rev. Lett. 116, 065501 (2016).

[82] Z. Budrikis, D. F. Castellanos, S. Sandfeld, M. Zaiser, and S. Zapperi, Nat. Commun. 8, 15928 (2017).

[83] T. Vojta, J. Phys. A: Math. Gen. 39, R143 (2006).

[84] K. A. Dahmen, Y. Ben-Zion, and J. T. Uhl, Phys. Rev. Lett. 102, 175501 (2009). 
[85] M. Ovaska, A. Lehtinen, M. J. Alava, L. Laurson, and S. Zapperi, Phys. Rev. Lett. 119, 265501 (2017).

[86] J. Lin, T. Gueudré, A. Rosso, and M. Wyart, Phys. Rev. Lett. 115, 168001 (2015).

[87] J. Lin and M. Wyart, Phys. Rev. X 6, 011005 (2016).

[88] S. Karmakar, E. Lerner, and I. Procaccia, Phys. Rev. E: Stat. Nonlin. Soft Matter Phys. 82, 055103(R) (2010).

[89] D. S. Fisher, Phys. Rep. 301, 113 (1998).

[90] B. Bakó, I. Groma, G. Györgyi, and G. T. Zimányi, Phys. Rev. Lett. 98, 075701 (2007).

[91] H. G. E. Hentschel, P. K. Jaiswal, I. Procaccia, and S. Sastry, Phys. Rev. E 92, 062302 (2015).

[92] D. Zhang, K. A. Dahmen, and M. Ostoja-Starzewski, Phys. Rev. E 95, 032902 (2017).

[93] U. F. Kocks and H. Mecking, Prog. Mater. Sci. 48, 171 (2003).

[94] F. J. Perez-Reche, C. Triguero, G. Zanzotto, and L. Truskinovsky, Phys. Rev. B 94, 144102 (2016).

[95] A. Tang, H. Liu, G. Liu, Y. Zhong, L. Wang, Q. Lu, J. Wang, and Y. Shen, Phys. Rev. Lett. 124, 155501 (2020).
[96] S. R. Shenoy and T. Lookman, Phys. Rev. B 78, 144103 (2008).

[97] A. Treibergs, A. Cherkaev, and P. Krtolica, Int. J. Solids Struct. 184, 248 (2020).

[98] V. Kucher, X. Markenscoff, and M. Paukshto, J. Elast. 74, 135 (2004).

[99] S. Suresh, Fatigue of Materials (Cambridge University Press, Cambridge, 1998).

[100] G. Durin and S. Zapperi, J. Stat. Mech. (2006) P01002.

[101] M. Ozawa, L. Berthier, G. Biroli, and G. Tarjus, Phys. Rev. Res. 2, 023203 (2020).

[102] H. Bhaumik, G. Foffi, and S. Sastry, arXiv:1911.12957 (2019).

[103] S. Franz and J. Rocchi, J. Phys. A: Math. Theor. (2020), doi:10.1088/1751-8121/ab9aeb.

[104] R. Baggio, E. Arbib, P. Biscari, S. Conti, L. Truskinovsky, G. Zanzotto, and O. U. Salman, Phys. Rev. Lett. 123, 205501 (2019). 